\title{
Ruthenium Arene Derivatives with PN Hemilabile Ligands. P-C Cleavage and Phosphine to Phosphinite Transformation
}

\author{
Agustín Caballero a , Félix A. Jalón ${ }^{\text {a, }}$, Blanca R. Manzano ${ }^{a}$, Gustavo Espino ${ }^{b}$, \\ Mercedes Pérez ${ }^{\mathrm{b}}$, Antonio Mucientes ${ }^{\mathrm{c}}$, Francisco J. Poblete ${ }^{\mathrm{c}}$, Miguel Maestro ${ }^{\mathrm{d}}$. \\ ${ }^{a}$ Departamento de Química Inorgánica, Orgánica y Bioquímica. Facultad de \\ Químicas. Universidad de Castilla-La Mancha. Avda. Camilo J. Cela 10, 13071 \\ Ciudad Real, Spain.
}

${ }^{b}$ Departamento de Química. Facultad de Químicas y CyTA. Plaza Misael Bañuelos s/n, Burgos, Spain.

${ }^{c}$ Departamento de Química Física. . Facultad de Químicas. Universidad de CastillaLa Mancha. Avda. Camilo J. Cela 10, 13071 Ciudad Real, Spain.

${ }^{d}$ Departamento de Química Fundamental. Facultade de Ciencias, Universidade da Coruña, Campus da Zapateira, 15071, Spain. 


\section{S U P PL E M E N T A R Y M A T E R I A L FOR CRYSTAL STRUCTURE}

Table S1 - Crystal Data and Structure Refinement for 1a (GRU61), 1b (GRU93), 4a (GRU152) and 4b (GRU142).

Table S2 - Atomic coordinates ( x 10 $)$ and equivalent isotropic displacement parameters $\left(\AA^{2}\right.$ x $\left.10^{3}\right)$ for $1 \mathbf{a}$.

Table S3 - Atomic coordinates ( x 10 $)$ and equivalent isotropic displacement parameters $\left(\AA^{2} \times 10^{3}\right)$ for $\mathbf{1 b .}$

Table S4 - Atomic coordinates ( x 10 $)$ and equivalent isotropic displacement parameters $\left(\AA^{2} \times 10^{3}\right)$ for $\mathbf{4 a}$

Table S5 - Atomic coordinates $\left(\right.$ x $\left.10^{4}\right)$ and equivalent isotropic displacement parameters $\left(\AA^{2} \times 10^{3}\right)$ for $\mathbf{4 b}$.

Table S6 - Bond lengths ( $(\AA)$ and angles (degrees) for $\mathbf{1 a}$.

Table S7 - Bond lengths ( $)$ and angles (degrees) for $\mathbf{1 b}$.

Table S9 - Bond lengths ( $\AA$ ) and angles (degrees) for $\mathbf{4 a}$.

Table S8 - Bond lengths ( $(\AA)$ and angles (degrees) for $\mathbf{4 b}$.

Table S10 - Anisotropic Displacement Parameters $\left(\AA^{2}\right.$ x $\left.10^{3}\right)$ for $\mathbf{1 a}$

Table S11 - Anisotropic Displacement Parameters $\left(\AA^{2}\right.$ x $\left.10^{3}\right)$ for $\mathbf{1 b}$

Table S12 - Anisotropic Displacement Parameters $\left(\AA^{2} \times 10^{3}\right)$ for $\mathbf{4 a}$

Table S13 - Anisotropic Displacement Parameters $\left(\AA^{2} \times 10^{3}\right)$ for $\mathbf{4 b}$

Table S14 - Hydrogen coordinates (x $\left.10^{4}\right)$ and isotropic displacement parameters $\left(\AA^{2} \times 10^{3}\right)$ for $\mathbf{1 a}$

Table S15 - Hydrogen coordinates (x $\left.10^{4}\right)$ and isotropic displacement parameters $\left(\AA^{2} \times 10^{3}\right)$ for $\mathbf{1 b}$

Table S16 - Hydrogen coordinates (x $\left.10^{4}\right)$ and isotropic displacement parameters $\left(\AA^{2} \times 10^{3}\right)$ for $4 \mathbf{a}$ 
Table S17 - Hydrogen coordinates (x $\left.10^{4}\right)$ and isotropic displacement parameters $\left(\AA^{2} \times 10^{3}\right)$ for $\mathbf{4 b}$

Figure S1 - ORTEP drawing of 1a

Figure S2 - ORTEP drawing of $\mathbf{1 b}$

Figure S3 - ORTEP drawing of $\mathbf{4 a}$

Figure S4 - ORTEP drawing of $\mathbf{4 b}$ 


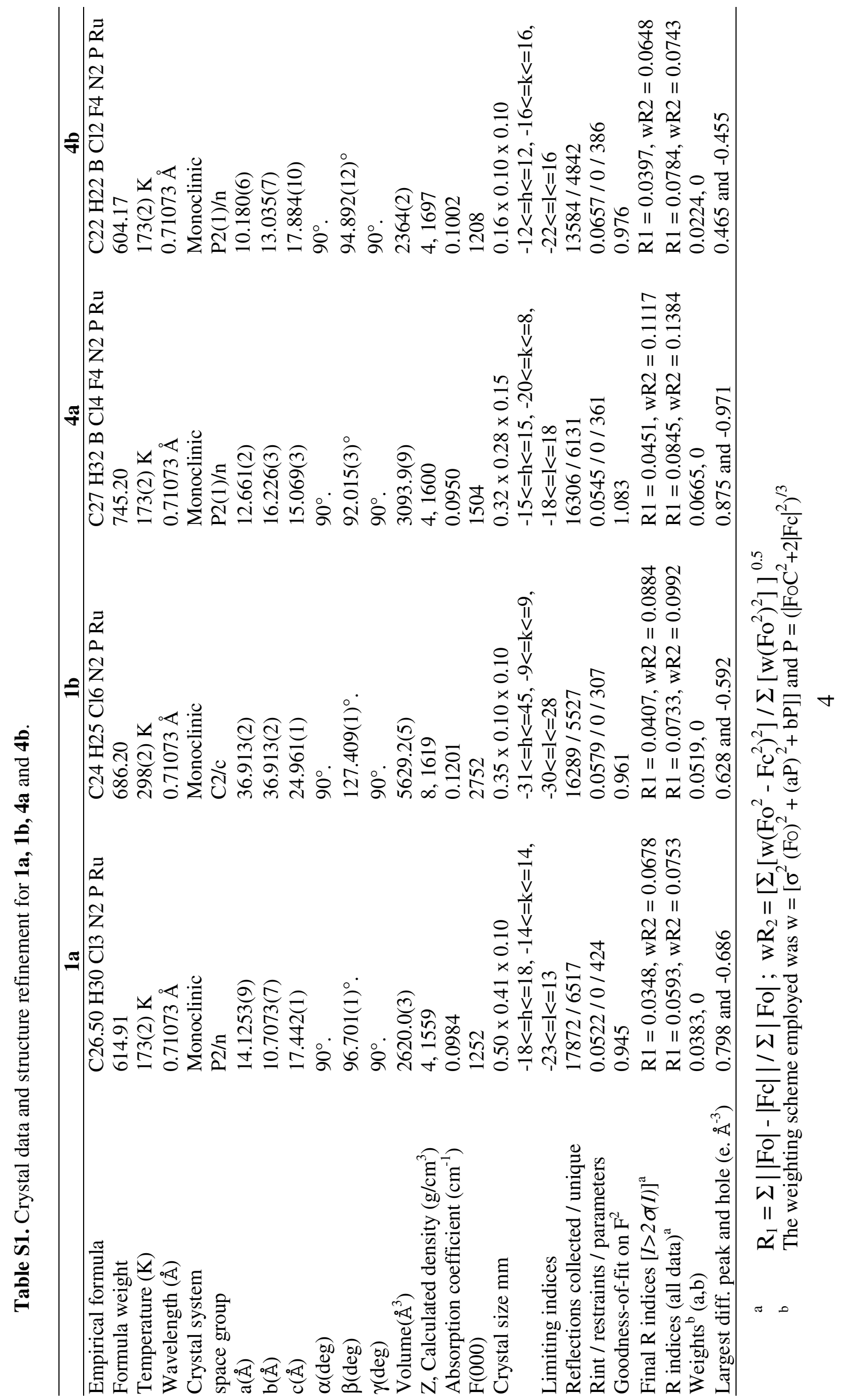


Table S2. Atomic coordinates $\left(\times 10^{4}\right)$ and equivalent isotropic displacement parameters $\left(\AA^{2} \times 10^{3}\right)$ for 1a. U(eq) is defined as one third of the trace of the orthogonalized Uij tensor.

\begin{tabular}{lcccc}
\hline & $\mathrm{X}$ & $\mathrm{y}$ & $\mathrm{Z}$ & $\mathrm{U}(\mathrm{eq})$ \\
\hline $\mathrm{Ru}(1)$ & $8304(1)$ & $2093(1)$ & $10856(1)$ & $17(1)$ \\
$\mathrm{Cl}(1)$ & $8527(1)$ & $4321(1)$ & $10842(1)$ & $26(1)$ \\
$\mathrm{Cl}(2)$ & $6694(1)$ & $2306(1)$ & $11204(1)$ & $25(1)$ \\
$\mathrm{P}(1)$ & $7585(1)$ & $2244(1)$ & $9573(1)$ & $17(1)$ \\
$\mathrm{N}(1)$ & $5774(2)$ & $963(2)$ & $9235(1)$ & $24(1)$ \\
$\mathrm{N}(2)$ & $7090(2)$ & $-197(2)$ & $9268(1)$ & $26(1)$ \\
$\mathrm{C}(1)$ & $9871(2)$ & $1971(2)$ & $11247(2)$ & $23(1)$ \\
$\mathrm{C}(2)$ & $9361(2)$ & $1799(3)$ & $11904(2)$ & $26(1)$ \\
$\mathrm{C}(3)$ & $8646(2)$ & $896(3)$ & $11894(2)$ & $26(1)$ \\
$\mathrm{C}(4)$ & $8387(2)$ & $117(3)$ & $11238(2)$ & $26(1)$ \\
$\mathrm{C}(5)$ & $8886(2)$ & $300(2)$ & $10589(2)$ & $24(1)$ \\
$\mathrm{C}(6)$ & $9629(2)$ & $1202(2)$ & $10599(2)$ & $23(1)$ \\
$\mathrm{C}(7)$ & $10661(2)$ & $2940(3)$ & $11299(2)$ & $27(1)$ \\
$\mathrm{C}(8)$ & $11503(2)$ & $2456(4)$ & $11850(2)$ & $39(1)$ \\
$\mathrm{C}(9)$ & $11000(3)$ & $3308(4)$ & $10534(2)$ & $43(1)$ \\
$\mathrm{C}(10)$ & $7604(2)$ & $-832(3)$ & $11238(2)$ & $34(1)$ \\
$\mathrm{C}(11)$ & $6751(2)$ & $957(2)$ & $9328(2)$ & $20(1)$ \\
$\mathrm{C}(12)$ & $5493(2)$ & $-253(3)$ & $9095(2)$ & $31(1)$ \\
$\mathrm{C}(13)$ & $6296(2)$ & $-938(3)$ & $9113(2)$ & $32(1)$ \\
$\mathrm{C}(14)$ & $5118(2)$ & $2000(3)$ & $9305(2)$ & $33(1)$ \\
$\mathrm{C}(15)$ & $8451(2)$ & $2096(2)$ & $8875(2)$ & $21(1)$ \\
$\mathrm{C}(16)$ & $8460(2)$ & $1148(3)$ & $8326(2)$ & $23(1)$ \\
$\mathrm{C}(17)$ & $9184(2)$ & $1116(3)$ & $7844(2)$ & $30(1)$ \\
$\mathrm{C}(18)$ & $9887(2)$ & $2010(3)$ & $7904(2)$ & $29(1)$ \\
$\mathrm{C}(19)$ & $9885(2)$ & $2954(3)$ & $8445(2)$ & $30(1)$ \\
$\mathrm{C}(20)$ & $9165(2)$ & $3010(3)$ & $8922(2)$ & $27(1)$ \\
$\mathrm{C}(21)$ & $6917(2)$ & $3641(2)$ & $9224(2)$ & $20(1)$ \\
$\mathrm{C}(22)$ & $6533(2)$ & $4462(3)$ & $9725(2)$ & $26(1)$ \\
$\mathrm{C}(23)$ & $5957(2)$ & $5439(3)$ & $9428(2)$ & $35(1)$ \\
$\mathrm{C}(24)$ & $5766(3)$ & $5606(3)$ & $8641(2)$ & $42(1)$ \\
$\mathrm{C}(25)$ & $6161(3)$ & $4817(3)$ & $8141(2)$ & $39(1)$ \\
$\mathrm{C}(26)$ & $6738(2)$ & $3830(3)$ & $8427(2)$ & $29(1)$ \\
$\mathrm{C}(1 \mathrm{~S})$ & 2500 & $4966(4)$ & 7500 & $86(1)$ \\
$\mathrm{Cl}(1 \mathrm{~S})$ & $1598(1)$ & $4042(1)$ & $7016(1)$ & \\
\hline & & & & \\
\hline
\end{tabular}


Table S3. Atomic coordinates $\left(\times 10^{4}\right)$ and equivalent isotropic displacement parameters $\left(\AA^{2} \times 10^{3}\right)$ for $\mathbf{1 b}$. $\mathrm{U}(\mathrm{eq})$ is defined as one third of the trace of the orthogonalized Uij tensor.

\begin{tabular}{|c|c|c|c|c|}
\hline & $X$ & $\mathrm{y}$ & $\mathrm{Z}$ & $\mathrm{U}(\mathrm{eq})$ \\
\hline $\mathrm{Ru}(1)$ & $1718(1)$ & $3554(1)$ & 4931(1) & $32(1)$ \\
\hline $\mathrm{Cl}(1)$ & $1990(1)$ & $6234(1)$ & $4796(1)$ & $46(1)$ \\
\hline $\mathrm{Cl}(2)$ & 2411(1) & $3492(1)$ & $6072(1)$ & $45(1)$ \\
\hline $\mathrm{P}(1)$ & $1362(1)$ & $5217(1)$ & $5282(1)$ & $32(1)$ \\
\hline $\mathrm{N}(1)$ & $1146(1)$ & $2667(4)$ & $5876(2)$ & $44(1)$ \\
\hline $\mathrm{N}(2)$ & $551(1)$ & $4140(5)$ & $5042(2)$ & $46(1)$ \\
\hline $\mathrm{C}(1)$ & $1731(2)$ & $720(6)$ & $4939(3)$ & $69(2)$ \\
\hline $\mathrm{C}(2)$ & $1940(2)$ & $1230(6)$ & $4641(3)$ & $64(2)$ \\
\hline $\mathrm{C}(3)$ & $1713(2)$ & $2357(6)$ & $4106(3)$ & $60(1)$ \\
\hline$C(4)$ & $1282(2)$ & 2996(6) & $3846(2)$ & $59(1)$ \\
\hline$C(5)$ & $1069(2)$ & $2450(7)$ & $4117(3)$ & $65(2)$ \\
\hline $\mathrm{C}(6)$ & $1291(2)$ & $1279(7)$ & $4665(3)$ & $70(2)$ \\
\hline$C(7)$ & $995(1)$ & $3931(5)$ & $5400(2)$ & $37(1)$ \\
\hline $\mathrm{C}(8)$ & $774(2)$ & $2076(6)$ & $5808(3)$ & $55(1)$ \\
\hline $\mathrm{C}(9)$ & $413(2)$ & 2992(6) & $5300(3)$ & $56(1)$ \\
\hline $\mathrm{C}(10)$ & $1608(2)$ & $2042(7)$ & $6371(3)$ & $69(2)$ \\
\hline $\mathrm{C}(11)$ & 1711(1) & $6434(5)$ & $6078(2)$ & $36(1)$ \\
\hline $\mathrm{C}(12)$ & $1607(2)$ & $6498(6)$ & $6524(2)$ & $48(1)$ \\
\hline $\mathrm{C}(13)$ & $1872(2)$ & $7461(6)$ & $7111(2)$ & $56(1)$ \\
\hline $\mathrm{C}(14)$ & $2234(2)$ & $8418(6)$ & $7248(2)$ & $54(1)$ \\
\hline $\mathrm{C}(15)$ & $2336(1)$ & $8386(6)$ & $6806(2)$ & $51(1)$ \\
\hline C(16) & $2085(1)$ & $7383(5)$ & $6230(2)$ & $40(1)$ \\
\hline $\mathrm{C}(17)$ & $943(1)$ & $6818(5)$ & $4675(2)$ & $36(1)$ \\
\hline $\mathrm{C}(18)$ & $702(1)$ & $7829(5)$ & $4819(2)$ & $46(1)$ \\
\hline C(19) & $361(2)$ & $8952(5)$ & 4331(3) & $57(1)$ \\
\hline $\mathrm{C}(20)$ & $265(2)$ & $9083(6)$ & $3714(3)$ & $64(1)$ \\
\hline $\mathrm{C}(21)$ & $508(2)$ & $8126(6)$ & $3565(3)$ & $63(1)$ \\
\hline $\mathrm{C}(22)$ & $845(1)$ & $6998(6)$ & $4046(2)$ & $47(1)$ \\
\hline $\mathrm{C}(1 \mathrm{~S})$ & $339(2)$ & $5326(8)$ & $6538(3)$ & $77(2)$ \\
\hline $\mathrm{C}(2 \mathrm{~S})$ & $1639(2)$ & $1876(7)$ & $8249(3)$ & $86(2)$ \\
\hline $\mathrm{Cl}(1 \mathrm{~S})$ & $617(1)$ & $7079(3)$ & $6484(1)$ & 111(1) \\
\hline $\mathrm{Cl}(2 \mathrm{~S})$ & 231(1) & $5782(3)$ & 7113(1) & $133(1)$ \\
\hline $\mathrm{Cl}(3 \mathrm{~S})$ & $1540(1)$ & $3922(2)$ & $7875(1)$ & $103(1)$ \\
\hline $\mathrm{Cl}(4 \mathrm{~S})$ & 1201(1) & $443(3)$ & 7709(1) & 141(1) \\
\hline
\end{tabular}


Table S4. Atomic coordinates $\left(\times 10^{4}\right)$ and equivalent isotropic displacement parameters $\left(\AA^{2} \times 10^{3}\right)$ for $4 \mathbf{a}$. $U(\mathrm{eq})$ is defined as one third of the trace of the orthogonalized Uij tensor.

\begin{tabular}{|c|c|c|c|c|}
\hline & $\mathrm{X}$ & $\mathrm{y}$ & $\mathrm{Z}$ & $\mathrm{U}(\mathrm{eq})$ \\
\hline $\mathrm{Ru}(1)$ & $7244(1)$ & $4769(1)$ & $8532(1)$ & $18(1)$ \\
\hline $\mathrm{P}(1)$ & $8370(1)$ & $5844(1)$ & $8170(1)$ & $18(1)$ \\
\hline $\mathrm{Cl}(1)$ & $7887(1)$ & $4053(1)$ & $7254(1)$ & $25(1)$ \\
\hline $\mathrm{Cl}(2)$ & $8710(1)$ & $4230(1)$ & $9405(1)$ & $25(1)$ \\
\hline $\mathrm{N}(1)$ & $10022(3)$ & $4786(3)$ & $7704(3)$ & $25(1)$ \\
\hline $\mathrm{N}(2)$ & $10618(3)$ & $6007(2)$ & $7916(3)$ & $22(1)$ \\
\hline $\mathrm{C}(1)$ & $6334(4)$ & $4945(3)$ & $9731(3)$ & $28(1)$ \\
\hline $\mathrm{C}(2)$ & $6157(4)$ & $4120(3)$ & $9440(3)$ & $30(1)$ \\
\hline $\mathrm{C}(3)$ & $5807(4)$ & $3975(3)$ & $8551(3)$ & $26(1)$ \\
\hline$C(4)$ & $5607(3)$ & $4644(3)$ & $7937(3)$ & 21(1) \\
\hline $\mathrm{C}(5)$ & $5755(4)$ & $5450(3)$ & $8265(3)$ & $23(1)$ \\
\hline$C(6)$ & $6123(4)$ & $5594(3)$ & $9149(3)$ & $26(1)$ \\
\hline $\mathrm{C}(7)$ & $6758(4)$ & $5088(4)$ & $10674(3)$ & $38(2)$ \\
\hline $\mathrm{C}(8)$ & $5229(4)$ & $4448(3)$ & $7014(3)$ & $30(1)$ \\
\hline $\mathrm{C}(9)$ & $4038(4)$ & $4299(4)$ & $7000(4)$ & $50(2)$ \\
\hline $\mathrm{C}(10)$ & $5511(5)$ & $5097(4)$ & $6329(4)$ & $42(2)$ \\
\hline $\mathrm{C}(11)$ & $8553(3)$ & $6626(3)$ & $9041(3)$ & $18(1)$ \\
\hline $\mathrm{C}(12)$ & $8413(4)$ & $7462(3)$ & $8888(4)$ & $27(1)$ \\
\hline $\mathrm{C}(13)$ & $8551(4)$ & $8018(3)$ & $9585(3)$ & $29(1)$ \\
\hline$C(14)$ & $8829(4)$ & $7739(3)$ & 10424(3) & $28(1)$ \\
\hline $\mathrm{C}(15)$ & $8968(4)$ & $6902(3)$ & $10576(3)$ & $27(1)$ \\
\hline $\mathrm{C}(16)$ & $8830(4)$ & $6348(3)$ & $9887(3)$ & $23(1)$ \\
\hline $\mathrm{C}(17)$ & 7993(4) & $6363(3)$ & $7130(3)$ & $23(1)$ \\
\hline C(18) & $7129(4)$ & $6901(3)$ & $7108(3)$ & $26(1)$ \\
\hline C(19) & $6763(4)$ & $7234(3)$ & $6321(4)$ & $32(1)$ \\
\hline $\mathrm{C}(20)$ & $7237(5)$ & $7030(4)$ & $5546(4)$ & $43(2)$ \\
\hline $\mathrm{C}(21)$ & $8071(5)$ & $6480(4)$ & $5541(3)$ & $38(1)$ \\
\hline $\mathrm{C}(22)$ & $8460(4)$ & $6146(3)$ & $6345(3)$ & $32(1)$ \\
\hline $\mathrm{C}(23)$ & $9739(4)$ & $5543(3)$ & $7945(3)$ & $20(1)$ \\
\hline $\mathrm{C}(24)$ & $11076(4)$ & $4760(3)$ & $7508(3)$ & $28(1)$ \\
\hline $\mathrm{C}(25)$ & $11443(4)$ & $5532(3)$ & $7643(3)$ & $29(1)$ \\
\hline $\mathrm{C}(26)$ & $10737(4)$ & $6885(3)$ & $8138(4)$ & $40(2)$ \\
\hline $\mathrm{C}(1 \mathrm{~S})$ & $5860(6)$ & $8259(4)$ & $9842(4)$ & $63(2)$ \\
\hline $\mathrm{Cl}(1 \mathrm{~S})$ & $6033(2)$ & $7589(1)$ & $10745(1)$ & $74(1)$ \\
\hline $\mathrm{Cl}(2 \mathrm{~S})$ & $6081(2)$ & $9263(1)$ & $10164(2)$ & $100(1)$ \\
\hline $\mathrm{B}(1)$ & $3893(5)$ & $7339(4)$ & $8087(5)$ & $36(2)$ \\
\hline $\mathrm{F}(1)$ & $4741(3)$ & $7725(3)$ & $7747(3)$ & $75(1)$ \\
\hline $\mathrm{F}(2)$ & $3098(4)$ & $7894(3)$ & $8109(4)$ & $117(2)$ \\
\hline $\mathrm{F}(3)$ & $3596(3)$ & $6666(3)$ & $7593(2)$ & $66(1)$ \\
\hline $\mathrm{F}(4)$ & $4150(5)$ & $7082(3)$ & $8926(3)$ & $111(2)$ \\
\hline
\end{tabular}


Table S5. Atomic coordinates $\left(\times 10^{4}\right)$ and equivalent isotropic displacement parameters $\left(\AA^{2} \times 10^{3}\right)$ for $\mathbf{4 b}$. $U(e q)$ is defined as one third of the trace of the orthogonalized Uij tensor.

\begin{tabular}{|c|c|c|c|c|}
\hline & $\mathrm{X}$ & $\mathrm{y}$ & $\mathrm{Z}$ & $\mathrm{U}(\mathrm{eq})$ \\
\hline $\mathrm{Ru}(1)$ & $3491(1)$ & $3945(1)$ & $1043(1)$ & $18(1)$ \\
\hline $\mathrm{Cl}(1)$ & 2154(1) & $4695(1)$ & $17(1)$ & $25(1)$ \\
\hline $\mathrm{Cl}(2)$ & $4335(1)$ & $5654(1)$ & $1319(1)$ & $29(1)$ \\
\hline $\mathrm{P}(1)$ & $1737(1)$ & $4223(1)$ & $1784(1)$ & $18(1)$ \\
\hline $\mathrm{N}(1)$ & $1427(4)$ & $6236(2)$ & $1291(2)$ & $27(1)$ \\
\hline $\mathrm{N}(2)$ & $-397(3)$ & $5720(2)$ & $1684(2)$ & $21(1)$ \\
\hline $\mathrm{C}(1)$ & $3478(6)$ & $2339(4)$ & $1340(4)$ & $51(2)$ \\
\hline$C(2)$ & $3383(5)$ & $2412(4)$ & $564(3)$ & $44(1)$ \\
\hline $\mathrm{C}(3)$ & $4346(6)$ & $2939(4)$ & $209(3)$ & $42(1)$ \\
\hline$C(4)$ & $5358(5)$ & $3412(4)$ & $615(3)$ & $43(1)$ \\
\hline$C(5)$ & $5444(5)$ & $3358(4)$ & $1402(3)$ & $49(2)$ \\
\hline$C(6)$ & $4532(6)$ & $2816(4)$ & $1769(3)$ & $47(2)$ \\
\hline$C(7)$ & $2117(4)$ & $4336(3)$ & $2800(2)$ & $19(1)$ \\
\hline $\mathrm{C}(8)$ & $3377(4)$ & $4618(3)$ & $3068(2)$ & $22(1)$ \\
\hline $\mathrm{C}(9)$ & $3688(5)$ & $4788(3)$ & $3826(2)$ & $26(1)$ \\
\hline$C(10)$ & $2723(5)$ & $4677(3)$ & $4322(2)$ & $29(1)$ \\
\hline$C(11)$ & $1477(5)$ & $4393(4)$ & $4065(2)$ & $35(1)$ \\
\hline$C(12)$ & $1142(4)$ & $4221(3)$ & $3304(2)$ & $30(1)$ \\
\hline$C(13)$ & $460(4)$ & $3272(3)$ & $1606(2)$ & $17(1)$ \\
\hline$C(14)$ & $244(4)$ & 2481(3) & $2094(2)$ & $24(1)$ \\
\hline$C(15)$ & $-734(4)$ & $1770(3)$ & $1922(2)$ & $28(1)$ \\
\hline$C(16)$ & $-1515(4)$ & $1839(3)$ & $1261(2)$ & $29(1)$ \\
\hline$C(17)$ & $-1296(5)$ & $2609(3)$ & $756(3)$ & $29(1)$ \\
\hline$C(18)$ & $-306(4)$ & $3323(3)$ & $922(2)$ & $24(1)$ \\
\hline$C(19)$ & $839(4)$ & $5431(3)$ & $1584(2)$ & $19(1)$ \\
\hline$C(20)$ & $-578(5)$ & $6710(3)$ & $1431(2)$ & $26(1)$ \\
\hline$C(21)$ & $558(5)$ & $7029(3)$ & 1191(2) & $28(1)$ \\
\hline$C(22)$ & $-1452(6)$ & $5165(4)$ & $2014(3)$ & $36(1)$ \\
\hline $\mathrm{B}(1)$ & $7560(5)$ & $8959(4)$ & $1331(3)$ & $26(1)$ \\
\hline $\mathrm{F}(1)$ & $8396(2)$ & $8741(2)$ & $779(1)$ & $34(1)$ \\
\hline $\mathrm{F}(2)$ & $6720(2)$ & $9753(2)$ & $1105(1)$ & $36(1)$ \\
\hline $\mathrm{F}(3)$ & $6834(2)$ & $8096(2)$ & $1475(1)$ & $44(1)$ \\
\hline $\mathrm{F}(4)$ & $8333(3)$ & $9244(2)$ & $1980(1)$ & $46(1)$ \\
\hline
\end{tabular}


Table S6. Bond lengths $[\AA]$ and angles [deg] for $\mathbf{1 a}$.

\begin{tabular}{|c|c|c|c|}
\hline $\mathrm{Ru}(1)-\mathrm{C}(5)$ & $2.161(3)$ & $\mathrm{C}(10)-\mathrm{H}(10 \mathrm{~B})$ & $0.98(4)$ \\
\hline $\mathrm{Ru}(1)-\mathrm{C}(6)$ & $2.193(3)$ & $\mathrm{C}(10)-\mathrm{H}(10 \mathrm{C})$ & $0.96(4)$ \\
\hline $\mathrm{Ru}(1)-\mathrm{C}(4)$ & $2.218(3)$ & $C(12)-C(13)$ & $1.348(4)$ \\
\hline $\mathrm{Ru}(1)-\mathrm{C}(3)$ & $2.225(3)$ & $\mathrm{C}(12)-\mathrm{H}(12)$ & $0.91(3)$ \\
\hline $\mathrm{Ru}(1)-\mathrm{C}(1)$ & $2.243(3)$ & $\mathrm{C}(13)-\mathrm{H}(13)$ & $0.94(3)$ \\
\hline $\mathrm{Ru}(1)-\mathrm{C}(2)$ & $2.243(3)$ & $\mathrm{C}(14)-\mathrm{H}(14 \mathrm{~A})$ & $0.99(4)$ \\
\hline $\mathrm{Ru}(1)-\mathrm{P}(1)$ & $2.3523(7)$ & $\mathrm{C}(14)-\mathrm{H}(14 \mathrm{~B})$ & $0.98(4)$ \\
\hline $\mathrm{Ru}(1)-\mathrm{Cl}(1)$ & $2.4070(7)$ & $\mathrm{C}(14)-\mathrm{H}(14 \mathrm{C})$ & $0.97(4)$ \\
\hline $\mathrm{Ru}(1)-\mathrm{Cl}(2)$ & $2.4320(7)$ & $C(15)-C(16)$ & $1.396(4)$ \\
\hline $\mathrm{P}(1)-\mathrm{C}(15)$ & $1.831(3)$ & $C(15)-C(20)$ & $1.401(4)$ \\
\hline $\mathrm{P}(1)-\mathrm{C}(11)$ & $1.831(3)$ & $C(16)-C(17)$ & $1.399(4)$ \\
\hline $\mathrm{P}(1)-\mathrm{C}(21)$ & $1.835(3)$ & $\mathrm{C}(16)-\mathrm{H}(16)$ & $0.95(3)$ \\
\hline $\mathrm{N}(1)-\mathrm{C}(11)$ & $1.371(3)$ & $\mathrm{C}(17)-\mathrm{C}(18)$ & $1.375(4)$ \\
\hline $\mathrm{N}(1)-\mathrm{C}(12)$ & $1.374(4)$ & $\mathrm{C}(17)-\mathrm{H}(17)$ & $0.93(3)$ \\
\hline $\mathrm{N}(1)-\mathrm{C}(14)$ & $1.460(4)$ & $\mathrm{C}(18)-\mathrm{C}(19)$ & $1.383(4)$ \\
\hline $\mathrm{N}(2)-\mathrm{C}(11)$ & $1.334(3)$ & $\mathrm{C}(18)-\mathrm{H}(18)$ & $0.90(3)$ \\
\hline $\mathrm{N}(2)-\mathrm{C}(13)$ & $1.375(4)$ & $C(19)-C(20)$ & $1.388(4)$ \\
\hline$C(1)-C(6)$ & $1.408(4)$ & $\mathrm{C}(19)-\mathrm{H}(19)$ & $0.91(4)$ \\
\hline$C(1)-C(2)$ & $1.434(4)$ & $\mathrm{C}(20)-\mathrm{H}(20)$ & $0.97(3)$ \\
\hline $\mathrm{C}(1)-\mathrm{C}(7)$ & $1.519(4)$ & $\mathrm{C}(21)-\mathrm{C}(22)$ & $1.394(4)$ \\
\hline$C(2)-C(3)$ & $1.397(4)$ & $C(21)-C(26)$ & $1.397(4)$ \\
\hline $\mathrm{C}(2)-\mathrm{H}(2)$ & $0.89(3)$ & $\mathrm{C}(22)-\mathrm{C}(23)$ & $1.388(4)$ \\
\hline$C(3)-C(4)$ & $1.428(4)$ & $\mathrm{C}(22)-\mathrm{H}(22)$ & $0.90(3)$ \\
\hline $\mathrm{C}(3)-\mathrm{H}(3)$ & $0.95(3)$ & $C(23)-C(24)$ & $1.379(5)$ \\
\hline$C(4)-C(5)$ & $1.416(4)$ & $\mathrm{C}(23)-\mathrm{H}(23)$ & $0.99(3)$ \\
\hline C(4)-C(10) & $1.503(4)$ & $\mathrm{C}(24)-\mathrm{C}(25)$ & $1.378(5)$ \\
\hline$C(5)-C(6)$ & $1.425(4)$ & $\mathrm{C}(24)-\mathrm{H}(24)$ & $0.90(4)$ \\
\hline $\mathrm{C}(5)-\mathrm{H}(5)$ & $1.00(3)$ & $C(25)-C(26)$ & $1.392(4)$ \\
\hline $\mathrm{C}(6)-\mathrm{H}(6)$ & $0.93(3)$ & $\mathrm{C}(25)-\mathrm{H}(25)$ & $1.02(4)$ \\
\hline$C(7)-C(9)$ & $1.520(5)$ & $\mathrm{C}(26)-\mathrm{H}(26)$ & $0.98(3)$ \\
\hline$C(7)-C(8)$ & $1.529(4)$ & $\mathrm{C}(1 \mathrm{~S})-\mathrm{Cl}(1 \mathrm{~S})$ & $1.751(3)$ \\
\hline $\mathrm{C}(7)-\mathrm{H}(7)$ & $1.02(3)$ & $\mathrm{C}(1 \mathrm{~S})-\mathrm{Cl}(1 \mathrm{~S}) \# 1$ & $1.751(3)$ \\
\hline $\mathrm{C}(8)-\mathrm{H}(8 \mathrm{~A})$ & $0.99(3)$ & $\mathrm{C}(1 \mathrm{~S})-\mathrm{H}(1 \mathrm{~A})$ & $1.00(3)$ \\
\hline $\mathrm{C}(8)-\mathrm{H}(8 \mathrm{~B})$ & $0.97(3)$ & & \\
\hline $\mathrm{C}(8)-\mathrm{H}(8 \mathrm{C})$ & $0.92(4)$ & $\mathrm{C}(5)-\mathrm{Ru}(1)-\mathrm{C}(6)$ & $38.20(10)$ \\
\hline $\mathrm{C}(9)-\mathrm{H}(9 \mathrm{~A})$ & $0.95(3)$ & $\mathrm{C}(5)-\mathrm{Ru}(1)-\mathrm{C}(4)$ & $37.71(10)$ \\
\hline $\mathrm{C}(9)-\mathrm{H}(9 \mathrm{~B})$ & $0.92(4)$ & $\mathrm{C}(6)-\mathrm{Ru}(1)-\mathrm{C}(4)$ & $68.28(10)$ \\
\hline $\mathrm{C}(9)-\mathrm{H}(9 \mathrm{C})$ & $1.02(4)$ & $\mathrm{C}(5)-\mathrm{Ru}(1)-\mathrm{C}(3)$ & $67.16(11)$ \\
\hline C(10)-H(10A) & $0.98(4)$ & $\mathrm{C}(6)-\mathrm{Ru}(1)-\mathrm{C}(3)$ & $78.84(11)$ \\
\hline
\end{tabular}




\begin{tabular}{|c|c|c|c|}
\hline $\mathrm{C}(4)-\mathrm{Ru}(1)-\mathrm{C}(3)$ & $37.51(11)$ & $C(6)-C(1)-C(2)$ & $118.0(2)$ \\
\hline $\mathrm{C}(5)-\mathrm{Ru}(1)-\mathrm{C}(1)$ & $68.11(10)$ & $C(6)-C(1)-C(7)$ & $123.7(3)$ \\
\hline $\mathrm{C}(6)-\mathrm{Ru}(1)-\mathrm{C}(1)$ & $37.01(10)$ & $\mathrm{C}(2)-\mathrm{C}(1)-\mathrm{C}(7)$ & $118.3(2)$ \\
\hline $\mathrm{C}(4)-\mathrm{Ru}(1)-\mathrm{C}(1)$ & $80.63(10)$ & $\mathrm{C}(6)-\mathrm{C}(1)-\mathrm{Ru}(1)$ & $69.57(15)$ \\
\hline $\mathrm{C}(3)-\mathrm{Ru}(1)-\mathrm{C}(1)$ & $66.79(10)$ & $\mathrm{C}(2)-\mathrm{C}(1)-\mathrm{Ru}(1)$ & $71.38(15)$ \\
\hline $\mathrm{C}(5)-\mathrm{Ru}(1)-\mathrm{C}(2)$ & $79.48(11)$ & $\mathrm{C}(7)-\mathrm{C}(1)-\mathrm{Ru}(1)$ & $132.12(18)$ \\
\hline $\mathrm{C}(6)-\mathrm{Ru}(1)-\mathrm{C}(2)$ & $66.60(11)$ & $\mathrm{C}(3)-\mathrm{C}(2)-\mathrm{C}(1)$ & $120.6(3)$ \\
\hline $\mathrm{C}(4)-\mathrm{Ru}(1)-\mathrm{C}(2)$ & $67.32(11)$ & $\mathrm{C}(3)-\mathrm{C}(2)-\mathrm{Ru}(1)$ & $71.06(16)$ \\
\hline $\mathrm{C}(3)-\mathrm{Ru}(1)-\mathrm{C}(2)$ & $36.44(10)$ & $\mathrm{C}(1)-\mathrm{C}(2)-\mathrm{Ru}(1)$ & $71.34(15)$ \\
\hline $\mathrm{C}(1)-\mathrm{Ru}(1)-\mathrm{C}(2)$ & $37.29(10)$ & $\mathrm{C}(3)-\mathrm{C}(2)-\mathrm{H}(2)$ & $120(2)$ \\
\hline $\mathrm{C}(5)-\mathrm{Ru}(1)-\mathrm{P}(1)$ & $89.32(8)$ & $\mathrm{C}(1)-\mathrm{C}(2)-\mathrm{H}(2)$ & $119(2)$ \\
\hline $\mathrm{C}(6)-\mathrm{Ru}(1)-\mathrm{P}(1)$ & $96.82(8)$ & $\mathrm{Ru}(1)-\mathrm{C}(2)-\mathrm{H}(2)$ & $127(2)$ \\
\hline $\mathrm{C}(4)-\mathrm{Ru}(1)-\mathrm{P}(1)$ & $110.68(8)$ & $\mathrm{C}(2)-\mathrm{C}(3)-\mathrm{C}(4)$ & $122.1(3)$ \\
\hline $\mathrm{C}(3)-\mathrm{Ru}(1)-\mathrm{P}(1)$ & $147.37(8)$ & $\mathrm{C}(2)-\mathrm{C}(3)-\mathrm{Ru}(1)$ & $72.50(16)$ \\
\hline $\mathrm{C}(1)-\mathrm{Ru}(1)-\mathrm{P}(1)$ & $126.46(7)$ & $\mathrm{C}(4)-\mathrm{C}(3)-\mathrm{Ru}(1)$ & $70.98(16)$ \\
\hline $\mathrm{C}(2)-\mathrm{Ru}(1)-\mathrm{P}(1)$ & $163.11(8)$ & $\mathrm{C}(2)-\mathrm{C}(3)-\mathrm{H}(3)$ & $116.8(16)$ \\
\hline $\mathrm{C}(5)-\mathrm{Ru}(1)-\mathrm{Cl}(1)$ & $145.60(8)$ & $\mathrm{C}(4)-\mathrm{C}(3)-\mathrm{H}(3)$ & $120.8(16)$ \\
\hline $\mathrm{C}(6)-\mathrm{Ru}(1)-\mathrm{Cl}(1)$ & $108.26(7)$ & $\mathrm{Ru}(1)-\mathrm{C}(3)-\mathrm{H}(3)$ & $125.0(16)$ \\
\hline $\mathrm{C}(4)-\mathrm{Ru}(1)-\mathrm{Cl}(1)$ & $161.17(8)$ & $C(5)-C(4)-C(3)$ & $117.1(2)$ \\
\hline $\mathrm{C}(3)-\mathrm{Ru}(1)-\mathrm{Cl}(1)$ & $124.27(8)$ & $C(5)-C(4)-C(10)$ & $122.0(3)$ \\
\hline $\mathrm{C}(1)-\mathrm{Ru}(1)-\mathrm{Cl}(1)$ & $86.29(7)$ & $C(3)-C(4)-C(10)$ & $121.0(3)$ \\
\hline $\mathrm{C}(2)-\mathrm{Ru}(1)-\mathrm{Cl}(1)$ & $94.12(8)$ & $\mathrm{C}(5)-\mathrm{C}(4)-\mathrm{Ru}(1)$ & $68.97(15)$ \\
\hline $\mathrm{P}(1)-\mathrm{Ru}(1)-\mathrm{Cl}(1)$ & $87.99(2)$ & $\mathrm{C}(3)-\mathrm{C}(4)-\mathrm{Ru}(1)$ & $71.51(16)$ \\
\hline $\mathrm{C}(5)-\mathrm{Ru}(1)-\mathrm{Cl}(2)$ & $121.84(7)$ & $\mathrm{C}(10)-\mathrm{C}(4)-\mathrm{Ru}(1)$ & $129.2(2)$ \\
\hline $\mathrm{C}(6)-\mathrm{Ru}(1)-\mathrm{Cl}(2)$ & $159.56(7)$ & $C(4)-C(5)-C(6)$ & 121.2(3) \\
\hline $\mathrm{C}(4)-\mathrm{Ru}(1)-\mathrm{Cl}(2)$ & $91.89(7)$ & $\mathrm{C}(4)-\mathrm{C}(5)-\mathrm{Ru}(1)$ & $73.32(15)$ \\
\hline $\mathrm{C}(3)-\mathrm{Ru}(1)-\mathrm{Cl}(2)$ & $88.40(8)$ & $C(6)-C(5)-R u(1)$ & $72.11(15)$ \\
\hline $\mathrm{C}(1)-\mathrm{Ru}(1)-\mathrm{Cl}(2)$ & $148.03(7)$ & $\mathrm{C}(4)-\mathrm{C}(5)-\mathrm{H}(5)$ & $118.6(15)$ \\
\hline $\mathrm{C}(2)-\mathrm{Ru}(1)-\mathrm{Cl}(2)$ & $111.31(8)$ & $\mathrm{C}(6)-\mathrm{C}(5)-\mathrm{H}(5)$ & $120.0(14)$ \\
\hline $\mathrm{P}(1)-\mathrm{Ru}(1)-\mathrm{Cl}(2)$ & $85.32(2)$ & $\mathrm{Ru}(1)-\mathrm{C}(5)-\mathrm{H}(5)$ & $123.7(14)$ \\
\hline $\mathrm{Cl}(1)-\mathrm{Ru}(1)-\mathrm{Cl}(2)$ & $92.10(2)$ & $C(1)-C(6)-C(5)$ & $121.1(3)$ \\
\hline $\mathrm{C}(15)-\mathrm{P}(1)-\mathrm{C}(11)$ & $103.99(12)$ & $C(1)-C(6)-R u(1)$ & $73.42(15)$ \\
\hline $\mathrm{C}(15)-\mathrm{P}(1)-\mathrm{C}(21)$ & $101.92(12)$ & $C(5)-C(6)-R u(1)$ & $69.69(14)$ \\
\hline $\mathrm{C}(11)-\mathrm{P}(1)-\mathrm{C}(21)$ & $104.11(12)$ & $\mathrm{C}(1)-\mathrm{C}(6)-\mathrm{H}(6)$ & $117.6(19)$ \\
\hline $\mathrm{C}(15)-\mathrm{P}(1)-\mathrm{Ru}(1)$ & $112.30(9)$ & $\mathrm{C}(5)-\mathrm{C}(6)-\mathrm{H}(6)$ & $120.8(19)$ \\
\hline $\mathrm{C}(11)-\mathrm{P}(1)-\mathrm{Ru}(1)$ & 111.41(9) & $\mathrm{Ru}(1)-\mathrm{C}(6)-\mathrm{H}(6)$ & $122.6(18)$ \\
\hline $\mathrm{C}(21)-\mathrm{P}(1)-\mathrm{Ru}(1)$ & $121.35(9)$ & $\mathrm{C}(1)-\mathrm{C}(7)-\mathrm{C}(9)$ & $115.4(3)$ \\
\hline $\mathrm{C}(11)-\mathrm{N}(1)-\mathrm{C}(12)$ & $106.4(2)$ & $\mathrm{C}(1)-\mathrm{C}(7)-\mathrm{C}(8)$ & $108.5(2)$ \\
\hline $\mathrm{C}(11)-\mathrm{N}(1)-\mathrm{C}(14)$ & $129.3(2)$ & $\mathrm{C}(9)-\mathrm{C}(7)-\mathrm{C}(8)$ & $109.8(3)$ \\
\hline $\mathrm{C}(12)-\mathrm{N}(1)-\mathrm{C}(14)$ & $124.2(2)$ & $\mathrm{C}(1)-\mathrm{C}(7)-\mathrm{H}(7)$ & $104.9(17)$ \\
\hline $\mathrm{C}(11)-\mathrm{N}(2)-\mathrm{C}(13)$ & $104.9(2)$ & $\mathrm{C}(9)-\mathrm{C}(7)-\mathrm{H}(7)$ & $108.7(18)$ \\
\hline
\end{tabular}




\begin{tabular}{|c|c|c|c|}
\hline $\mathrm{C}(8)-\mathrm{C}(7)-\mathrm{H}(7)$ & $109.4(17)$ & $C(15)-C(16)-C(17)$ & $119.7(3)$ \\
\hline $\mathrm{C}(7)-\mathrm{C}(8)-\mathrm{H}(8 \mathrm{~A})$ & $109(2)$ & $\mathrm{C}(15)-\mathrm{C}(16)-\mathrm{H}(16)$ & $120(2)$ \\
\hline $\mathrm{C}(7)-\mathrm{C}(8)-\mathrm{H}(8 \mathrm{~B})$ & $106(2)$ & $\mathrm{C}(17)-\mathrm{C}(16)-\mathrm{H}(16)$ & $120(2)$ \\
\hline $\mathrm{H}(8 \mathrm{~A})-\mathrm{C}(8)-\mathrm{H}(8 \mathrm{~B})$ & $109(3)$ & $\mathrm{C}(18)-\mathrm{C}(17)-\mathrm{C}(16)$ & $120.6(3)$ \\
\hline $\mathrm{C}(7)-\mathrm{C}(8)-\mathrm{H}(8 \mathrm{C})$ & $108(2)$ & $\mathrm{C}(18)-\mathrm{C}(17)-\mathrm{H}(17)$ & $123.3(19)$ \\
\hline $\mathrm{H}(8 \mathrm{~A})-\mathrm{C}(8)-\mathrm{H}(8 \mathrm{C})$ & $112(3)$ & $\mathrm{C}(16)-\mathrm{C}(17)-\mathrm{H}(17)$ & $116.1(19)$ \\
\hline $\mathrm{H}(8 \mathrm{~B})-\mathrm{C}(8)-\mathrm{H}(8 \mathrm{C})$ & $113(3)$ & $\mathrm{C}(17)-\mathrm{C}(18)-\mathrm{C}(19)$ & $120.1(3)$ \\
\hline $\mathrm{C}(7)-\mathrm{C}(9)-\mathrm{H}(9 \mathrm{~A})$ & $110(2)$ & $\mathrm{C}(17)-\mathrm{C}(18)-\mathrm{H}(18)$ & $118.7(19)$ \\
\hline $\mathrm{C}(7)-\mathrm{C}(9)-\mathrm{H}(9 \mathrm{~B})$ & $114(3)$ & $\mathrm{C}(19)-\mathrm{C}(18)-\mathrm{H}(18)$ & 121.1(19) \\
\hline $\mathrm{H}(9 \mathrm{~A})-\mathrm{C}(9)-\mathrm{H}(9 \mathrm{~B})$ & $112(3)$ & $\mathrm{C}(18)-\mathrm{C}(19)-\mathrm{C}(20)$ & $120.0(3)$ \\
\hline $\mathrm{C}(7)-\mathrm{C}(9)-\mathrm{H}(9 \mathrm{C})$ & $117(2)$ & $\mathrm{C}(18)-\mathrm{C}(19)-\mathrm{H}(19)$ & $124(2)$ \\
\hline $\mathrm{H}(9 \mathrm{~A})-\mathrm{C}(9)-\mathrm{H}(9 \mathrm{C})$ & $104(3)$ & $\mathrm{C}(20)-\mathrm{C}(19)-\mathrm{H}(19)$ & $115(2)$ \\
\hline $\mathrm{H}(9 \mathrm{~B})-\mathrm{C}(9)-\mathrm{H}(9 \mathrm{C})$ & $99(3)$ & $C(19)-C(20)-C(15)$ & $120.5(3)$ \\
\hline $\mathrm{C}(4)-\mathrm{C}(10)-\mathrm{H}(10 \mathrm{~A})$ & $109(2)$ & $\mathrm{C}(19)-\mathrm{C}(20)-\mathrm{H}(20)$ & $118.1(18)$ \\
\hline $\mathrm{C}(4)-\mathrm{C}(10)-\mathrm{H}(10 \mathrm{~B})$ & $113(2)$ & $\mathrm{C}(15)-\mathrm{C}(20)-\mathrm{H}(20)$ & $121.4(18)$ \\
\hline $\mathrm{H}(10 \mathrm{~A})-\mathrm{C}(10)-\mathrm{H}(10 \mathrm{~B})$ & $108(3)$ & $\mathrm{C}(22)-\mathrm{C}(21)-\mathrm{C}(26)$ & $119.6(3)$ \\
\hline $\mathrm{C}(4)-\mathrm{C}(10)-\mathrm{H}(10 \mathrm{C})$ & 111(2) & $\mathrm{C}(22)-\mathrm{C}(21)-\mathrm{P}(1)$ & $121.9(2)$ \\
\hline $\mathrm{H}(10 \mathrm{~A})-\mathrm{C}(10)-\mathrm{H}(10 \mathrm{C})$ & $103(3)$ & $\mathrm{C}(26)-\mathrm{C}(21)-\mathrm{P}(1)$ & $118.4(2)$ \\
\hline $\mathrm{H}(10 \mathrm{~B})-\mathrm{C}(10)-\mathrm{H}(10 \mathrm{C})$ & 111(3) & $\mathrm{C}(23)-\mathrm{C}(22)-\mathrm{C}(21)$ & $119.8(3)$ \\
\hline $\mathrm{N}(2)-\mathrm{C}(11)-\mathrm{N}(1)$ & 111.2(2) & $\mathrm{C}(23)-\mathrm{C}(22)-\mathrm{H}(22)$ & $120.8(19)$ \\
\hline $\mathrm{N}(2)-\mathrm{C}(11)-\mathrm{P}(1)$ & 119.2(2) & $\mathrm{C}(21)-\mathrm{C}(22)-\mathrm{H}(22)$ & $119.4(19)$ \\
\hline $\mathrm{N}(1)-\mathrm{C}(11)-\mathrm{P}(1)$ & $129.5(2)$ & $\mathrm{C}(24)-\mathrm{C}(23)-\mathrm{C}(22)$ & $120.4(3)$ \\
\hline $\mathrm{C}(13)-\mathrm{C}(12)-\mathrm{N}(1)$ & $106.7(3)$ & $\mathrm{C}(24)-\mathrm{C}(23)-\mathrm{H}(23)$ & $120.5(19)$ \\
\hline $\mathrm{C}(13)-\mathrm{C}(12)-\mathrm{H}(12)$ & $133.3(18)$ & $\mathrm{C}(22)-\mathrm{C}(23)-\mathrm{H}(23)$ & $119(2)$ \\
\hline $\mathrm{N}(1)-\mathrm{C}(12)-\mathrm{H}(12)$ & $120.0(18)$ & $\mathrm{C}(23)-\mathrm{C}(24)-\mathrm{C}(25)$ & $120.3(3)$ \\
\hline $\mathrm{C}(12)-\mathrm{C}(13)-\mathrm{N}(2)$ & $110.8(3)$ & $\mathrm{C}(23)-\mathrm{C}(24)-\mathrm{H}(24)$ & $121(2)$ \\
\hline $\mathrm{C}(12)-\mathrm{C}(13)-\mathrm{H}(13)$ & $127.4(19)$ & $\mathrm{C}(25)-\mathrm{C}(24)-\mathrm{H}(24)$ & $118(2)$ \\
\hline $\mathrm{N}(2)-\mathrm{C}(13)-\mathrm{H}(13)$ & $121.8(19)$ & $C(24)-C(25)-C(26)$ & $120.1(3)$ \\
\hline $\mathrm{N}(1)-\mathrm{C}(14)-\mathrm{H}(14 \mathrm{~A})$ & $109(2)$ & $\mathrm{C}(24)-\mathrm{C}(25)-\mathrm{H}(25)$ & $121.6(19)$ \\
\hline N(1)-C(14)-H(14B) & 111(2) & $\mathrm{C}(26)-\mathrm{C}(25)-\mathrm{H}(25)$ & $118.2(19)$ \\
\hline $\mathrm{H}(14 \mathrm{~A})-\mathrm{C}(14)-\mathrm{H}(14 \mathrm{~B})$ & $110(3)$ & $C(25)-C(26)-C(21)$ & $119.8(3)$ \\
\hline $\mathrm{N}(1)-\mathrm{C}(14)-\mathrm{H}(14 \mathrm{C})$ & 111(2) & $\mathrm{C}(25)-\mathrm{C}(26)-\mathrm{H}(26)$ & $119.2(19)$ \\
\hline $\mathrm{H}(14 \mathrm{~A})-\mathrm{C}(14)-\mathrm{H}(14 \mathrm{C})$ & $108(3)$ & $\mathrm{C}(21)-\mathrm{C}(26)-\mathrm{H}(26)$ & $121.0(19)$ \\
\hline $\mathrm{H}(14 \mathrm{~B})-\mathrm{C}(14)-\mathrm{H}(14 \mathrm{C})$ & $108(3)$ & $\mathrm{Cl}(1 \mathrm{~S})-\mathrm{C}(1 \mathrm{~S})-\mathrm{Cl}(1 \mathrm{~S}) \# 1$ & 111.1(3) \\
\hline$C(16)-C(15)-C(20)$ & $119.0(2)$ & $\mathrm{Cl}(1 \mathrm{~S})-\mathrm{C}(1 \mathrm{~S})-\mathrm{H}(1 \mathrm{~A})$ & 111(2) \\
\hline C(16)-C(15)-P(1) & $125.3(2)$ & $\mathrm{Cl}(1 \mathrm{~S}) \# 1-\mathrm{C}(1 \mathrm{~S})-\mathrm{H}(1 \mathrm{~A})$ & $108(2)$ \\
\hline$C(20)-C(15)-P(1)$ & $115.7(2)$ & & \\
\hline
\end{tabular}

Symmetry transformations used to generate equivalent atoms:

\#1 - $\mathrm{x}+1 / 2, \mathrm{y},-\mathrm{z}+3 / 2$ 
Table S7. Bond lengths $[\AA]$ and angles [deg] for $\mathbf{1 b}$.

\begin{tabular}{|c|c|c|c|}
\hline $\mathrm{Ru}(1)-\mathrm{C}(5)$ & $2.164(4)$ & $\mathrm{C}(1 \mathrm{~S})-\mathrm{Cl}(2 \mathrm{~S})$ & $1.740(5)$ \\
\hline $\mathrm{Ru}(1)-\mathrm{C}(6)$ & $2.172(4)$ & $\mathrm{C}(1 \mathrm{~S})-\mathrm{Cl}(1 \mathrm{~S})$ & $1.750(6)$ \\
\hline $\mathrm{Ru}(1)-\mathrm{C}(1)$ & $2.180(4)$ & $\mathrm{C}(2 \mathrm{~S})-\mathrm{Cl}(4 \mathrm{~S})$ & $1.732(6)$ \\
\hline $\mathrm{Ru}(1)-\mathrm{C}(4)$ & $2.195(5)$ & $\mathrm{C}(2 \mathrm{~S})-\mathrm{Cl}(3 \mathrm{~S})$ & $1.752(6)$ \\
\hline $\mathrm{Ru}(1)-\mathrm{C}(3)$ & $2.245(4)$ & & \\
\hline $\mathrm{Ru}(1)-\mathrm{C}(2)$ & $2.260(4)$ & $\mathrm{C}(5)-\mathrm{Ru}(1)-\mathrm{C}(6)$ & $38.0(2)$ \\
\hline $\mathrm{Ru}(1)-\mathrm{P}(1)$ & $2.3529(10)$ & $\mathrm{C}(5)-\mathrm{Ru}(1)-\mathrm{C}(1)$ & $67.7(2)$ \\
\hline $\mathrm{Ru}(1)-\mathrm{Cl}(1)$ & $2.4055(10)$ & $\mathrm{C}(6)-\mathrm{Ru}(1)-\mathrm{C}(1)$ & $37.3(2)$ \\
\hline $\mathrm{Ru}(1)-\mathrm{Cl}(2)$ & $2.4085(11)$ & $\mathrm{C}(5)-\mathrm{Ru}(1)-\mathrm{C}(4)$ & $36.86(19)$ \\
\hline$P(1)-C(17)$ & $1.832(4)$ & $\mathrm{C}(6)-\mathrm{Ru}(1)-\mathrm{C}(4)$ & $67.2(2)$ \\
\hline $\mathrm{P}(1)-\mathrm{C}(11)$ & $1.837(4)$ & $\mathrm{C}(1)-\mathrm{Ru}(1)-\mathrm{C}(4)$ & $79.08(19)$ \\
\hline $\mathrm{P}(1)-\mathrm{C}(7)$ & $1.838(4)$ & $\mathrm{C}(5)-\mathrm{Ru}(1)-\mathrm{C}(3)$ & $65.81(19)$ \\
\hline $\mathrm{N}(1)-\mathrm{C}(8)$ & $1.357(5)$ & $\mathrm{C}(6)-\mathrm{Ru}(1)-\mathrm{C}(3)$ & $77.82(18)$ \\
\hline $\mathrm{N}(1)-\mathrm{C}(7)$ & $1.365(5)$ & $\mathrm{C}(1)-\mathrm{Ru}(1)-\mathrm{C}(3)$ & $65.6(2)$ \\
\hline $\mathrm{N}(1)-\mathrm{C}(10)$ & $1.451(5)$ & $\mathrm{C}(4)-\mathrm{Ru}(1)-\mathrm{C}(3)$ & $36.55(18)$ \\
\hline $\mathrm{N}(2)-\mathrm{C}(7)$ & $1.316(5)$ & $\mathrm{C}(5)-\mathrm{Ru}(1)-\mathrm{C}(2)$ & $78.37(18)$ \\
\hline $\mathrm{N}(2)-\mathrm{C}(9)$ & $1.361(5)$ & $\mathrm{C}(6)-\mathrm{Ru}(1)-\mathrm{C}(2)$ & $66.5(2)$ \\
\hline$C(1)-C(6)$ & $1.393(7)$ & $\mathrm{C}(1)-\mathrm{Ru}(1)-\mathrm{C}(2)$ & $37.08(19)$ \\
\hline $\mathrm{C}(1)-\mathrm{C}(2)$ & $1.414(7)$ & $\mathrm{C}(4)-\mathrm{Ru}(1)-\mathrm{C}(2)$ & $65.56(19)$ \\
\hline $\mathrm{C}(2)-\mathrm{C}(3)$ & $1.369(7)$ & $\mathrm{C}(3)-\mathrm{Ru}(1)-\mathrm{C}(2)$ & $35.38(19)$ \\
\hline$C(3)-C(4)$ & $1.393(7)$ & $\mathrm{C}(5)-\mathrm{Ru}(1)-\mathrm{P}(1)$ & $90.68(13)$ \\
\hline$C(4)-C(5)$ & $1.378(7)$ & $\mathrm{C}(6)-\mathrm{Ru}(1)-\mathrm{P}(1)$ & $94.90(14)$ \\
\hline $\mathrm{C}(5)-\mathrm{C}(6)$ & $1.411(8)$ & $\mathrm{C}(1)-\mathrm{Ru}(1)-\mathrm{P}(1)$ & $123.66(17)$ \\
\hline $\mathrm{C}(8)-\mathrm{C}(9)$ & $1.350(6)$ & $\mathrm{C}(4)-\mathrm{Ru}(1)-\mathrm{P}(1)$ & $114.13(14)$ \\
\hline$C(11)-C(12)$ & $1.379(5)$ & $\mathrm{C}(3)-\mathrm{Ru}(1)-\mathrm{P}(1)$ & $150.38(15)$ \\
\hline $\mathrm{C}(11)-\mathrm{C}(16)$ & $1.395(5)$ & $\mathrm{C}(2)-\mathrm{Ru}(1)-\mathrm{P}(1)$ & $160.47(15)$ \\
\hline $\mathrm{C}(12)-\mathrm{C}(13)$ & $1.384(6)$ & $\mathrm{C}(5)-\mathrm{Ru}(1)-\mathrm{Cl}(1)$ & $121.55(18)$ \\
\hline $\mathrm{C}(13)-\mathrm{C}(14)$ & $1.375(6)$ & $\mathrm{C}(6)-\mathrm{Ru}(1)-\mathrm{Cl}(1)$ & $159.21(18)$ \\
\hline $\mathrm{C}(14)-\mathrm{C}(15)$ & $1.365(6)$ & $\mathrm{C}(1)-\mathrm{Ru}(1)-\mathrm{Cl}(1)$ & $148.16(17)$ \\
\hline $\mathrm{C}(15)-\mathrm{C}(16)$ & $1.378(6)$ & $\mathrm{C}(4)-\mathrm{Ru}(1)-\mathrm{Cl}(1)$ & $92.87(14)$ \\
\hline $\mathrm{C}(17)-\mathrm{C}(18)$ & $1.384(5)$ & $\mathrm{C}(3)-\mathrm{Ru}(1)-\mathrm{Cl}(1)$ & 89.64(13) \\
\hline $\mathrm{C}(17)-\mathrm{C}(22)$ & $1.387(6)$ & $\mathrm{C}(2)-\mathrm{Ru}(1)-\mathrm{Cl}(1)$ & $111.66(15)$ \\
\hline $\mathrm{C}(18)-\mathrm{C}(19)$ & $1.398(6)$ & $\mathrm{P}(1)-\mathrm{Ru}(1)-\mathrm{Cl}(1)$ & $87.84(4)$ \\
\hline C(19)-C(20) & $1.357(7)$ & $\mathrm{C}(5)-\mathrm{Ru}(1)-\mathrm{Cl}(2)$ & $151.35(18)$ \\
\hline $\mathrm{C}(20)-\mathrm{C}(21)$ & $1.374(7)$ & $\mathrm{C}(6)-\mathrm{Ru}(1)-\mathrm{Cl}(2)$ & $113.46(18)$ \\
\hline $\mathrm{C}(21)-\mathrm{C}(22)$ & $1.389(6)$ & $\mathrm{C}(1)-\mathrm{Ru}(1)-\mathrm{Cl}(2)$ & $88.20(15)$ \\
\hline
\end{tabular}




$\begin{array}{lclr}\mathrm{C}(4)-\mathrm{Ru}(1)-\mathrm{Cl}(2) & 155.73(14) & \mathrm{C}(4)-\mathrm{C}(5)-\mathrm{Ru}(1) & 72.8(3) \\ \mathrm{C}(3)-\mathrm{Ru}(1)-\mathrm{Cl}(2) & 119.21(15) & \mathrm{C}(6)-\mathrm{C}(5)-\mathrm{Ru}(1) & 71.3(3) \\ \mathrm{C}(2)-\mathrm{Ru}(1)-\mathrm{Cl}(2) & 91.90(14) & \mathrm{C}(1)-\mathrm{C}(6)-\mathrm{C}(5) & 119.4(5) \\ \mathrm{P}(1)-\mathrm{Ru}(1)-\mathrm{Cl}(2) & 90.13(4) & \mathrm{C}(1)-\mathrm{C}(6)-\mathrm{Ru}(1) & 71.6(3) \\ \mathrm{Cl}(1)-\mathrm{Ru}(1)-\mathrm{Cl}(2) & 87.10(4) & \mathrm{C}(5)-\mathrm{C}(6)-\mathrm{Ru}(1) & 70.7(3) \\ \mathrm{C}(17)-\mathrm{P}(1)-\mathrm{C}(11) & 103.75(17) & \mathrm{N}(2)-\mathrm{C}(7)-\mathrm{N}(1) & 111.1(3) \\ \mathrm{C}(17)-\mathrm{P}(1)-\mathrm{C}(7) & 99.27(17) & \mathrm{N}(2)-\mathrm{C}(7)-\mathrm{P}(1) & 124.0(3) \\ \mathrm{C}(11)-\mathrm{P}(1)-\mathrm{C}(7) & 101.65(18) & \mathrm{N}(1)-\mathrm{C}(7)-\mathrm{P}(1) & 124.8(3) \\ \mathrm{C}(17)-\mathrm{P}(1)-\mathrm{Ru}(1) & 116.02(13) & \mathrm{C}(9)-\mathrm{C}(8)-\mathrm{N}(1) & 106.8(4) \\ \mathrm{C}(11)-\mathrm{P}(1)-\mathrm{Ru}(1) & 119.76(12) & \mathrm{C}(8)-\mathrm{C}(9)-\mathrm{N}(2) & 110.1(4) \\ \mathrm{C}(7)-\mathrm{P}(1)-\mathrm{Ru}(1) & 113.53(12) & \mathrm{C}(12)-\mathrm{C}(11)-\mathrm{C}(16) & 118.4(4) \\ \mathrm{C}(8)-\mathrm{N}(1)-\mathrm{C}(7) & 106.4(3) & \mathrm{C}(12)-\mathrm{C}(11)-\mathrm{P}(1) & 122.3(3) \\ \mathrm{C}(8)-\mathrm{N}(1)-\mathrm{C}(10) & 125.0(4) & \mathrm{C}(16)-\mathrm{C}(11)-\mathrm{P}(1) & 119.2(3) \\ \mathrm{C}(7)-\mathrm{N}(1)-\mathrm{C}(10) & 128.6(3) & \mathrm{C}(11)-\mathrm{C}(12)-\mathrm{C}(13) & 120.7(4) \\ \mathrm{C}(7)-\mathrm{N}(2)-\mathrm{C}(9) & 105.6(4) & \mathrm{C}(14)-\mathrm{C}(13)-\mathrm{C}(12) & 120.3(4) \\ \mathrm{C}(6)-\mathrm{C}(1)-\mathrm{C}(2) & 120.1(5) & \mathrm{C}(15)-\mathrm{C}(14)-\mathrm{C}(13) & 119.5(4) \\ \mathrm{C}(6)-\mathrm{C}(1)-\mathrm{Ru}(1) & 71.0(3) & \mathrm{C}(14)-\mathrm{C}(15)-\mathrm{C}(16) & 120.8(4) \\ \mathrm{C}(2)-\mathrm{C}(1)-\mathrm{Ru}(1) & 74.5(3) & \mathrm{C}(15)-\mathrm{C}(16)-\mathrm{C}(11) & 120.2(4) \\ \mathrm{C}(3)-\mathrm{C}(2)-\mathrm{C}(1) & 118.9(5) & \mathrm{C}(18)-\mathrm{C}(17)-\mathrm{C}(22) & 118.2(4) \\ \mathrm{C}(3)-\mathrm{C}(2)-\mathrm{Ru}(1) & 71.7(3) & \mathrm{C}(18)-\mathrm{C}(17)-\mathrm{P}(1) & 121.0(3) \\ \mathrm{C}(1)-\mathrm{C}(2)-\mathrm{Ru}(1) & 68.4(3) & \mathrm{C}(22)-\mathrm{C}(17)-\mathrm{P}(1) & 120.8(3) \\ \mathrm{C}(2)-\mathrm{C}(3)-\mathrm{C}(4) & 121.7(5) & \mathrm{C}(17)-\mathrm{C}(18)-\mathrm{C}(19) & 120.1(5) \\ \mathrm{C}(2)-\mathrm{C}(3)-\mathrm{Ru}(1) & 72.9(3) & \mathrm{C}(20)-\mathrm{C}(19)-\mathrm{C}(18) & 120.7(5) \\ \mathrm{C}(4)-\mathrm{C}(3)-\mathrm{Ru}(1) & 69.7(3) & \mathrm{C}(19)-\mathrm{C}(20)-\mathrm{C}(21) & 119.5(5) \\ \mathrm{C}(5)-\mathrm{C}(4)-\mathrm{C}(3) & 119.7(5) & \mathrm{C}(20)-\mathrm{C}(21)-\mathrm{C}(22) & 110.3(3) \\ \mathrm{C}(5)-\mathrm{C}(4)-\mathrm{Ru}(1) & 70.3(3) & \mathrm{C}(17)-\mathrm{C}(22)-\mathrm{C}(21) & 11.4(3) \\ \mathrm{C}(3)-\mathrm{C}(4)-\mathrm{Ru}(1) & 73.7(3) & \mathrm{Cl}(2 \mathrm{~S})-\mathrm{C}(1 \mathrm{~S})-\mathrm{Cl}(1 \mathrm{~S}) & \mathrm{Cl}(4 \mathrm{~S})-\mathrm{C}(2 \mathrm{~S})-\mathrm{Cl}(3 \mathrm{~S}) \\ \mathrm{C}(4)-\mathrm{C}(5)-\mathrm{C}(6) & & & \\ & & & \end{array}$


Table S8. Bond lengths $[\AA]$ and angles [deg] for $\mathbf{4 a}$.

\begin{tabular}{|c|c|c|c|}
\hline $\mathrm{Ru}(1)-\mathrm{C}(6)$ & $2.183(5)$ & $\mathrm{C}(9)-\mathrm{H}(9 \mathrm{~B})$ & 0.9800 \\
\hline $\mathrm{Ru}(1)-\mathrm{C}(1)$ & $2.195(5)$ & $\mathrm{C}(9)-\mathrm{H}(9 \mathrm{C})$ & 0.9800 \\
\hline $\operatorname{Ru}(1)-C(5)$ & $2.210(5)$ & $\mathrm{C}(10)-\mathrm{H}(10 \mathrm{~A})$ & 0.9800 \\
\hline $\mathrm{Ru}(1)-\mathrm{C}(3)$ & $2.230(5)$ & $\mathrm{C}(10)-\mathrm{H}(10 \mathrm{~B})$ & 0.9800 \\
\hline $\mathrm{Ru}(1)-\mathrm{C}(2)$ & $2.237(5)$ & $\mathrm{C}(10)-\mathrm{H}(10 \mathrm{C})$ & 0.9800 \\
\hline $\mathrm{Ru}(1)-\mathrm{C}(4)$ & $2.238(5)$ & $\mathrm{C}(11)-\mathrm{C}(16)$ & $1.385(6)$ \\
\hline $\mathrm{Ru}(1)-\mathrm{P}(1)$ & $2.3304(13)$ & $\mathrm{C}(11)-\mathrm{C}(12)$ & $1.387(6)$ \\
\hline $\mathrm{Ru}(1)-\mathrm{Cl}(2)$ & $2.4012(13)$ & $\mathrm{C}(12)-\mathrm{C}(13)$ & 1.391(7) \\
\hline $\mathrm{Ru}(1)-\mathrm{Cl}(1)$ & $2.4148(12)$ & $\mathrm{C}(12)-\mathrm{H}(12 \mathrm{~A})$ & 0.9500 \\
\hline $\mathrm{P}(1)-\mathrm{C}(17)$ & $1.827(5)$ & $\mathrm{C}(13)-\mathrm{C}(14)$ & $1.377(7)$ \\
\hline $\mathrm{P}(1)-\mathrm{C}(11)$ & $1.834(5)$ & $\mathrm{C}(13)-\mathrm{H}(13 \mathrm{~A})$ & 0.9500 \\
\hline $\mathrm{P}(1)-\mathrm{C}(23)$ & $1.844(5)$ & $\mathrm{C}(14)-\mathrm{C}(15)$ & $1.387(7)$ \\
\hline $\mathrm{N}(1)-\mathrm{C}(23)$ & $1.333(6)$ & $\mathrm{C}(14)-\mathrm{H}(14 \mathrm{~A})$ & 0.9500 \\
\hline $\mathrm{N}(1)-\mathrm{C}(24)$ & $1.378(6)$ & $\mathrm{C}(15)-\mathrm{C}(16)$ & $1.380(6)$ \\
\hline $\mathrm{N}(1)-\mathrm{H}(1 \mathrm{~A})$ & 0.8800 & $\mathrm{C}(15)-\mathrm{H}(15 \mathrm{~A})$ & 0.9500 \\
\hline $\mathrm{N}(2)-\mathrm{C}(23)$ & $1.346(6)$ & $\mathrm{C}(16)-\mathrm{H}(16 \mathrm{~A})$ & 0.9500 \\
\hline $\mathrm{N}(2)-\mathrm{C}(25)$ & $1.373(6)$ & $\mathrm{C}(17)-\mathrm{C}(22)$ & $1.386(7)$ \\
\hline $\mathrm{N}(2)-\mathrm{C}(26)$ & $1.468(6)$ & $\mathrm{C}(17)-\mathrm{C}(18)$ & $1.399(7)$ \\
\hline$C(1)-C(6)$ & 1.391(7) & $\mathrm{C}(18)-\mathrm{C}(19)$ & $1.369(7)$ \\
\hline$C(1)-C(2)$ & $1.425(7)$ & $\mathrm{C}(18)-\mathrm{H}(18 \mathrm{~A})$ & 0.9500 \\
\hline $\mathrm{C}(1)-\mathrm{C}(7)$ & $1.519(7)$ & $\mathrm{C}(19)-\mathrm{C}(20)$ & $1.372(8)$ \\
\hline$C(2)-C(3)$ & $1.415(7)$ & $\mathrm{C}(19)-\mathrm{H}(19 \mathrm{~A})$ & 0.9500 \\
\hline $\mathrm{C}(2)-\mathrm{H}(2 \mathrm{~A})$ & 1.0000 & $\mathrm{C}(20)-\mathrm{C}(21)$ & $1.382(8)$ \\
\hline$C(3)-C(4)$ & $1.444(7)$ & $\mathrm{C}(20)-\mathrm{H}(20 \mathrm{~A})$ & 0.9500 \\
\hline $\mathrm{C}(3)-\mathrm{H}(3 \mathrm{~A})$ & 1.0000 & $\mathrm{C}(21)-\mathrm{C}(22)$ & $1.401(7)$ \\
\hline$C(4)-C(5)$ & $1.407(7)$ & $\mathrm{C}(21)-\mathrm{H}(21 \mathrm{~A})$ & 0.9500 \\
\hline$C(4)-C(8)$ & $1.490(7)$ & $\mathrm{C}(22)-\mathrm{H}(22 \mathrm{~A})$ & 0.9500 \\
\hline$C(5)-C(6)$ & $1.416(7)$ & $\mathrm{C}(24)-\mathrm{C}(25)$ & $1.348(7)$ \\
\hline $\mathrm{C}(5)-\mathrm{H}(5 \mathrm{~A})$ & 1.0000 & $\mathrm{C}(24)-\mathrm{H}(24 \mathrm{~A})$ & 0.9500 \\
\hline $\mathrm{C}(6)-\mathrm{H}(6 \mathrm{~A})$ & 1.0000 & $\mathrm{C}(25)-\mathrm{H}(25 \mathrm{~A})$ & 0.9500 \\
\hline $\mathrm{C}(7)-\mathrm{H}(7 \mathrm{~A})$ & 0.9800 & $\mathrm{C}(26)-\mathrm{H}(26 \mathrm{~A})$ & 0.9800 \\
\hline $\mathrm{C}(7)-\mathrm{H}(7 \mathrm{~B})$ & 0.9800 & $\mathrm{C}(26)-\mathrm{H}(26 \mathrm{~B})$ & 0.9800 \\
\hline $\mathrm{C}(7)-\mathrm{H}(7 \mathrm{C})$ & 0.9800 & $\mathrm{C}(26)-\mathrm{H}(26 \mathrm{C})$ & 0.9800 \\
\hline $\mathrm{C}(8)-\mathrm{C}(10)$ & $1.525(8)$ & $\mathrm{C}(1 \mathrm{~S})-\mathrm{Cl}(2 \mathrm{~S})$ & $1.721(7)$ \\
\hline $\mathrm{C}(8)-\mathrm{C}(9)$ & $1.528(7)$ & $\mathrm{C}(1 \mathrm{~S})-\mathrm{Cl}(1 \mathrm{~S})$ & $1.749(7)$ \\
\hline $\mathrm{C}(8)-\mathrm{H}(8 \mathrm{~A})$ & 1.0000 & $\mathrm{C}(1 \mathrm{~S})-\mathrm{H}(1 \mathrm{SA})$ & 0.9900 \\
\hline $\mathrm{C}(9)-\mathrm{H}(9 \mathrm{~A})$ & 0.9800 & $\mathrm{C}(1 \mathrm{~S})-\mathrm{H}(1 \mathrm{SB})$ & 0.9900 \\
\hline
\end{tabular}




\begin{tabular}{|c|c|c|c|}
\hline $\mathrm{B}(1)-\mathrm{F}(2)$ & $1.352(8)$ & $\mathrm{C}(4)-\mathrm{Ru}(1)-\mathrm{Cl}(1)$ & $88.31(13)$ \\
\hline $\mathrm{B}(1)-\mathrm{F}(1)$ & $1.360(7)$ & $\mathrm{P}(1)-\mathrm{Ru}(1)-\mathrm{Cl}(1)$ & $86.95(4)$ \\
\hline $\mathrm{B}(1)-\mathrm{F}(4)$ & $1.360(8)$ & $\mathrm{Cl}(2)-\mathrm{Ru}(1)-\mathrm{Cl}(1)$ & $89.22(4)$ \\
\hline \multirow[t]{2}{*}{$\mathrm{B}(1)-\mathrm{F}(3)$} & $1.367(8)$ & $\mathrm{C}(17)-\mathrm{P}(1)-\mathrm{C}(11)$ & $108.5(2)$ \\
\hline & & $\mathrm{C}(17)-\mathrm{P}(1)-\mathrm{C}(23)$ & $100.6(2)$ \\
\hline $\mathrm{C}(6)-\mathrm{Ru}(1)-\mathrm{C}(1)$ & $37.04(19)$ & $\mathrm{C}(11)-\mathrm{P}(1)-\mathrm{C}(23)$ & $102.7(2)$ \\
\hline $\mathrm{C}(6)-\mathrm{Ru}(1)-\mathrm{C}(5)$ & $37.59(17)$ & $\mathrm{C}(17)-\mathrm{P}(1)-\mathrm{Ru}(1)$ & $113.69(16)$ \\
\hline $\mathrm{C}(1)-\mathrm{Ru}(1)-\mathrm{C}(5)$ & $67.43(18)$ & $\mathrm{C}(11)-\mathrm{P}(1)-\mathrm{Ru}(1)$ & $114.40(15)$ \\
\hline $\mathrm{C}(6)-\mathrm{Ru}(1)-\mathrm{C}(3)$ & 78.81(19) & $\mathrm{C}(23)-\mathrm{P}(1)-\mathrm{Ru}(1)$ & $115.58(16)$ \\
\hline $\mathrm{C}(1)-\mathrm{Ru}(1)-\mathrm{C}(3)$ & $67.22(19)$ & $\mathrm{C}(23)-\mathrm{N}(1)-\mathrm{C}(24)$ & $111.0(4)$ \\
\hline $\mathrm{C}(5)-\mathrm{Ru}(1)-\mathrm{C}(3)$ & $66.43(19)$ & $\mathrm{C}(23)-\mathrm{N}(1)-\mathrm{H}(1 \mathrm{~A})$ & 124.5 \\
\hline $\mathrm{C}(6)-\mathrm{Ru}(1)-\mathrm{C}(2)$ & $66.8(2)$ & $\mathrm{C}(24)-\mathrm{N}(1)-\mathrm{H}(1 \mathrm{~A})$ & 124.5 \\
\hline $\mathrm{C}(1)-\mathrm{Ru}(1)-\mathrm{C}(2)$ & $37.48(19)$ & $\mathrm{C}(23)-\mathrm{N}(2)-\mathrm{C}(25)$ & $109.5(4)$ \\
\hline $\mathrm{C}(5)-\mathrm{Ru}(1)-\mathrm{C}(2)$ & 78.93(19) & $\mathrm{C}(23)-\mathrm{N}(2)-\mathrm{C}(26)$ & $127.8(4)$ \\
\hline $\mathrm{C}(3)-\mathrm{Ru}(1)-\mathrm{C}(2)$ & $36.94(18)$ & $\mathrm{C}(25)-\mathrm{N}(2)-\mathrm{C}(26)$ & $122.7(4)$ \\
\hline $\mathrm{C}(6)-\mathrm{Ru}(1)-\mathrm{C}(4)$ & 67.61(18) & $\mathrm{C}(6)-\mathrm{C}(1)-\mathrm{C}(2)$ & $119.5(5)$ \\
\hline $\mathrm{C}(1)-\mathrm{Ru}(1)-\mathrm{C}(4)$ & $80.57(18)$ & $C(6)-C(1)-C(7)$ & $121.9(5)$ \\
\hline $\mathrm{C}(5)-\mathrm{Ru}(1)-\mathrm{C}(4)$ & $36.87(17)$ & $\mathrm{C}(2)-\mathrm{C}(1)-\mathrm{C}(7)$ & $118.6(5)$ \\
\hline $\mathrm{C}(3)-\mathrm{Ru}(1)-\mathrm{C}(4)$ & $37.70(17)$ & $\mathrm{C}(6)-\mathrm{C}(1)-\mathrm{Ru}(1)$ & $71.0(3)$ \\
\hline $\mathrm{C}(2)-\mathrm{Ru}(1)-\mathrm{C}(4)$ & $67.78(18)$ & $\mathrm{C}(2)-\mathrm{C}(1)-\mathrm{Ru}(1)$ & $72.8(3)$ \\
\hline $\mathrm{C}(6)-\mathrm{Ru}(1)-\mathrm{P}(1)$ & 93.01(14) & $\mathrm{C}(7)-\mathrm{C}(1)-\mathrm{Ru}(1)$ & $127.7(4)$ \\
\hline $\mathrm{C}(1)-\mathrm{Ru}(1)-\mathrm{P}(1)$ & 115.98(15) & $\mathrm{C}(3)-\mathrm{C}(2)-\mathrm{C}(1)$ & $119.2(5)$ \\
\hline $\mathrm{C}(5)-\mathrm{Ru}(1)-\mathrm{P}(1)$ & $96.19(13)$ & $\mathrm{C}(3)-\mathrm{C}(2)-\mathrm{Ru}(1)$ & $71.3(3)$ \\
\hline $\mathrm{C}(3)-\mathrm{Ru}(1)-\mathrm{P}(1)$ & $160.33(14)$ & $\mathrm{C}(1)-\mathrm{C}(2)-\mathrm{Ru}(1)$ & $69.7(3)$ \\
\hline $\mathrm{C}(2)-\mathrm{Ru}(1)-\mathrm{P}(1)$ & $152.89(14)$ & $\mathrm{C}(3)-\mathrm{C}(2)-\mathrm{H}(2 \mathrm{~A})$ & 119.7 \\
\hline $\mathrm{C}(4)-\mathrm{Ru}(1)-\mathrm{P}(1)$ & $122.64(13)$ & $\mathrm{C}(1)-\mathrm{C}(2)-\mathrm{H}(2 \mathrm{~A})$ & 119.7 \\
\hline $\mathrm{C}(6)-\mathrm{Ru}(1)-\mathrm{Cl}(2)$ & $119.44(14)$ & $\mathrm{Ru}(1)-\mathrm{C}(2)-\mathrm{H}(2 \mathrm{~A})$ & 119.7 \\
\hline $\mathrm{C}(1)-\mathrm{Ru}(1)-\mathrm{Cl}(2)$ & $90.82(14)$ & $C(2)-C(3)-C(4)$ & $121.6(5)$ \\
\hline $\mathrm{C}(5)-\mathrm{Ru}(1)-\mathrm{Cl}(2)$ & 156.89(13) & $\mathrm{C}(2)-\mathrm{C}(3)-\mathrm{Ru}(1)$ & $71.8(3)$ \\
\hline $\mathrm{C}(3)-\mathrm{Ru}(1)-\mathrm{Cl}(2)$ & $113.46(14)$ & $\mathrm{C}(4)-\mathrm{C}(3)-\mathrm{Ru}(1)$ & $71.5(3)$ \\
\hline $\mathrm{C}(2)-\mathrm{Ru}(1)-\mathrm{Cl}(2)$ & $88.51(14)$ & $\mathrm{C}(2)-\mathrm{C}(3)-\mathrm{H}(3 \mathrm{~A})$ & 118.6 \\
\hline $\mathrm{C}(4)-\mathrm{Ru}(1)-\mathrm{Cl}(2)$ & $150.90(13)$ & $\mathrm{C}(4)-\mathrm{C}(3)-\mathrm{H}(3 \mathrm{~A})$ & 118.6 \\
\hline $\mathrm{P}(1)-\mathrm{Ru}(1)-\mathrm{Cl}(2)$ & $86.15(4)$ & $\mathrm{Ru}(1)-\mathrm{C}(3)-\mathrm{H}(3 \mathrm{~A})$ & 118.6 \\
\hline $\mathrm{C}(6)-\mathrm{Ru}(1)-\mathrm{Cl}(1)$ & 151.29(14) & $\mathrm{C}(5)-\mathrm{C}(4)-\mathrm{C}(3)$ & 117.1(4) \\
\hline $\mathrm{C}(1)-\mathrm{Ru}(1)-\mathrm{Cl}(1)$ & $157.02(15)$ & $C(5)-C(4)-C(8)$ & $124.1(5)$ \\
\hline $\mathrm{C}(5)-\mathrm{Ru}(1)-\mathrm{Cl}(1)$ & 113.84(13) & $\mathrm{C}(3)-\mathrm{C}(4)-\mathrm{C}(8)$ & $118.8(4)$ \\
\hline $\mathrm{C}(3)-\mathrm{Ru}(1)-\mathrm{Cl}(1)$ & $91.77(13)$ & $\mathrm{C}(5)-\mathrm{C}(4)-\mathrm{Ru}(1)$ & $70.5(3)$ \\
\hline $\mathrm{C}(2)-\mathrm{Ru}(1)-\mathrm{Cl}(1)$ & $119.56(15)$ & $\mathrm{C}(3)-\mathrm{C}(4)-\mathrm{Ru}(1)$ & $70.8(3)$ \\
\hline
\end{tabular}




\begin{tabular}{|c|c|c|c|}
\hline $\mathrm{C}(8)-\mathrm{C}(4)-\mathrm{Ru}(1)$ & $131.0(3)$ & $\mathrm{C}(16)-\mathrm{C}(11)-\mathrm{P}(1)$ & 117.1(4) \\
\hline$C(4)-C(5)-C(6)$ & $121.3(5)$ & $\mathrm{C}(12)-\mathrm{C}(11)-\mathrm{P}(1)$ & $123.1(4)$ \\
\hline$C(4)-C(5)-R u(1)$ & $72.7(3)$ & $\mathrm{C}(11)-\mathrm{C}(12)-\mathrm{C}(13)$ & $119.7(5)$ \\
\hline $\mathrm{C}(6)-\mathrm{C}(5)-\mathrm{Ru}(1)$ & $70.2(3)$ & $\mathrm{C}(11)-\mathrm{C}(12)-\mathrm{H}(12 \mathrm{~A})$ & 120.1 \\
\hline $\mathrm{C}(4)-\mathrm{C}(5)-\mathrm{H}(5 \mathrm{~A})$ & 118.7 & $\mathrm{C}(13)-\mathrm{C}(12)-\mathrm{H}(12 \mathrm{~A})$ & 120.1 \\
\hline $\mathrm{C}(6)-\mathrm{C}(5)-\mathrm{H}(5 \mathrm{~A})$ & 118.7 & $\mathrm{C}(14)-\mathrm{C}(13)-\mathrm{C}(12)$ & $120.2(5)$ \\
\hline $\mathrm{Ru}(1)-\mathrm{C}(5)-\mathrm{H}(5 \mathrm{~A})$ & 118.7 & $\mathrm{C}(14)-\mathrm{C}(13)-\mathrm{H}(13 \mathrm{~A})$ & 119.9 \\
\hline$C(1)-C(6)-C(5)$ & $121.2(5)$ & $\mathrm{C}(12)-\mathrm{C}(13)-\mathrm{H}(13 \mathrm{~A})$ & 119.9 \\
\hline $\mathrm{C}(1)-\mathrm{C}(6)-\mathrm{Ru}(1)$ & $71.9(3)$ & $\mathrm{C}(13)-\mathrm{C}(14)-\mathrm{C}(15)$ & $120.0(5)$ \\
\hline $\mathrm{C}(5)-\mathrm{C}(6)-\mathrm{Ru}(1)$ & $72.2(3)$ & $\mathrm{C}(13)-\mathrm{C}(14)-\mathrm{H}(14 \mathrm{~A})$ & 120.0 \\
\hline $\mathrm{C}(1)-\mathrm{C}(6)-\mathrm{H}(6 \mathrm{~A})$ & 118.9 & $\mathrm{C}(15)-\mathrm{C}(14)-\mathrm{H}(14 \mathrm{~A})$ & 120.0 \\
\hline $\mathrm{C}(5)-\mathrm{C}(6)-\mathrm{H}(6 \mathrm{~A})$ & 118.9 & $C(16)-C(15)-C(14)$ & $120.1(5)$ \\
\hline $\mathrm{Ru}(1)-\mathrm{C}(6)-\mathrm{H}(6 \mathrm{~A})$ & 118.9 & $\mathrm{C}(16)-\mathrm{C}(15)-\mathrm{H}(15 \mathrm{~A})$ & 119.9 \\
\hline $\mathrm{C}(1)-\mathrm{C}(7)-\mathrm{H}(7 \mathrm{~A})$ & 109.5 & $\mathrm{C}(14)-\mathrm{C}(15)-\mathrm{H}(15 \mathrm{~A})$ & 119.9 \\
\hline $\mathrm{C}(1)-\mathrm{C}(7)-\mathrm{H}(7 \mathrm{~B})$ & 109.5 & $\mathrm{C}(15)-\mathrm{C}(16)-\mathrm{C}(11)$ & $120.1(5)$ \\
\hline $\mathrm{H}(7 \mathrm{~A})-\mathrm{C}(7)-\mathrm{H}(7 \mathrm{~B})$ & 109.5 & $\mathrm{C}(15)-\mathrm{C}(16)-\mathrm{H}(16 \mathrm{~A})$ & 119.9 \\
\hline $\mathrm{C}(1)-\mathrm{C}(7)-\mathrm{H}(7 \mathrm{C})$ & 109.5 & $\mathrm{C}(11)-\mathrm{C}(16)-\mathrm{H}(16 \mathrm{~A})$ & 119.9 \\
\hline $\mathrm{H}(7 \mathrm{~A})-\mathrm{C}(7)-\mathrm{H}(7 \mathrm{C})$ & 109.5 & $\mathrm{C}(22)-\mathrm{C}(17)-\mathrm{C}(18)$ & $119.6(5)$ \\
\hline $\mathrm{H}(7 \mathrm{~B})-\mathrm{C}(7)-\mathrm{H}(7 \mathrm{C})$ & 109.5 & $\mathrm{C}(22)-\mathrm{C}(17)-\mathrm{P}(1)$ & $120.6(4)$ \\
\hline $\mathrm{C}(4)-\mathrm{C}(8)-\mathrm{C}(10)$ & $114.2(5)$ & $\mathrm{C}(18)-\mathrm{C}(17)-\mathrm{P}(1)$ & $119.3(4)$ \\
\hline $\mathrm{C}(4)-\mathrm{C}(8)-\mathrm{C}(9)$ & $109.4(4)$ & $\mathrm{C}(19)-\mathrm{C}(18)-\mathrm{C}(17)$ & $120.6(5)$ \\
\hline $\mathrm{C}(10)-\mathrm{C}(8)-\mathrm{C}(9)$ & $110.7(5)$ & $\mathrm{C}(19)-\mathrm{C}(18)-\mathrm{H}(18 \mathrm{~A})$ & 119.7 \\
\hline $\mathrm{C}(4)-\mathrm{C}(8)-\mathrm{H}(8 \mathrm{~A})$ & 107.4 & $\mathrm{C}(17)-\mathrm{C}(18)-\mathrm{H}(18 \mathrm{~A})$ & 119.7 \\
\hline $\mathrm{C}(10)-\mathrm{C}(8)-\mathrm{H}(8 \mathrm{~A})$ & 107.4 & $\mathrm{C}(18)-\mathrm{C}(19)-\mathrm{C}(20)$ & $119.8(5)$ \\
\hline $\mathrm{C}(9)-\mathrm{C}(8)-\mathrm{H}(8 \mathrm{~A})$ & 107.4 & $\mathrm{C}(18)-\mathrm{C}(19)-\mathrm{H}(19 \mathrm{~A})$ & 120.1 \\
\hline $\mathrm{C}(8)-\mathrm{C}(9)-\mathrm{H}(9 \mathrm{~A})$ & 109.5 & $\mathrm{C}(20)-\mathrm{C}(19)-\mathrm{H}(19 \mathrm{~A})$ & 120.1 \\
\hline $\mathrm{C}(8)-\mathrm{C}(9)-\mathrm{H}(9 \mathrm{~B})$ & 109.5 & $\mathrm{C}(19)-\mathrm{C}(20)-\mathrm{C}(21)$ & $121.2(5)$ \\
\hline $\mathrm{H}(9 \mathrm{~A})-\mathrm{C}(9)-\mathrm{H}(9 \mathrm{~B})$ & 109.5 & $\mathrm{C}(19)-\mathrm{C}(20)-\mathrm{H}(20 \mathrm{~A})$ & 119.4 \\
\hline $\mathrm{C}(8)-\mathrm{C}(9)-\mathrm{H}(9 \mathrm{C})$ & 109.5 & $\mathrm{C}(21)-\mathrm{C}(20)-\mathrm{H}(20 \mathrm{~A})$ & 119.4 \\
\hline $\mathrm{H}(9 \mathrm{~A})-\mathrm{C}(9)-\mathrm{H}(9 \mathrm{C})$ & 109.5 & $\mathrm{C}(20)-\mathrm{C}(21)-\mathrm{C}(22)$ & $119.3(5)$ \\
\hline $\mathrm{H}(9 \mathrm{~B})-\mathrm{C}(9)-\mathrm{H}(9 \mathrm{C})$ & 109.5 & $\mathrm{C}(20)-\mathrm{C}(21)-\mathrm{H}(21 \mathrm{~A})$ & 120.3 \\
\hline $\mathrm{C}(8)-\mathrm{C}(10)-\mathrm{H}(10 \mathrm{~A})$ & 109.5 & $\mathrm{C}(22)-\mathrm{C}(21)-\mathrm{H}(21 \mathrm{~A})$ & 120.3 \\
\hline $\mathrm{C}(8)-\mathrm{C}(10)-\mathrm{H}(10 \mathrm{~B})$ & 109.5 & $\mathrm{C}(17)-\mathrm{C}(22)-\mathrm{C}(21)$ & $119.5(5)$ \\
\hline $\mathrm{H}(10 \mathrm{~A})-\mathrm{C}(10)-\mathrm{H}(10 \mathrm{~B})$ & 109.5 & $\mathrm{C}(17)-\mathrm{C}(22)-\mathrm{H}(22 \mathrm{~A})$ & 120.2 \\
\hline $\mathrm{C}(8)-\mathrm{C}(10)-\mathrm{H}(10 \mathrm{C})$ & 109.5 & $\mathrm{C}(21)-\mathrm{C}(22)-\mathrm{H}(22 \mathrm{~A})$ & 120.2 \\
\hline $\mathrm{H}(10 \mathrm{~A})-\mathrm{C}(10)-\mathrm{H}(10 \mathrm{C})$ & 109.5 & $\mathrm{~N}(1)-\mathrm{C}(23)-\mathrm{N}(2)$ & $106.0(4)$ \\
\hline $\mathrm{H}(10 \mathrm{~B})-\mathrm{C}(10)-\mathrm{H}(10 \mathrm{C})$ & 109.5 & $\mathrm{~N}(1)-\mathrm{C}(23)-\mathrm{P}(1)$ & $123.9(4)$ \\
\hline$C(16)-C(11)-C(12)$ & $119.9(5)$ & $\mathrm{N}(2)-\mathrm{C}(23)-\mathrm{P}(1)$ & $129.9(4)$ \\
\hline
\end{tabular}




$\begin{array}{llll}\mathrm{C}(25)-\mathrm{C}(24)-\mathrm{N}(1) & 105.7(4) & \mathrm{Cl}(2 \mathrm{~S})-\mathrm{C}(1 \mathrm{~S})-\mathrm{Cl}(1 \mathrm{~S}) & 110.7(4) \\ \mathrm{C}(25)-\mathrm{C}(24)-\mathrm{H}(24 \mathrm{~A}) & 127.2 & \mathrm{Cl}(2 \mathrm{~S})-\mathrm{C}(1 \mathrm{~S})-\mathrm{H}(1 \mathrm{SA}) & 109.5 \\ \mathrm{~N}(1)-\mathrm{C}(24)-\mathrm{H}(24 \mathrm{~A}) & 127.2 & \mathrm{Cl}(1 \mathrm{~S})-\mathrm{C}(1 \mathrm{~S})-\mathrm{H}(1 \mathrm{SA}) & 109.5 \\ \mathrm{C}(24)-\mathrm{C}(25)-\mathrm{N}(2) & 107.8(4) & \mathrm{Cl}(2 \mathrm{~S})-\mathrm{C}(1 \mathrm{~S})-\mathrm{H}(1 \mathrm{SB}) & 109.5 \\ \mathrm{C}(24)-\mathrm{C}(25)-\mathrm{H}(25 \mathrm{~A}) & 126.1 & \mathrm{Cl}(1 \mathrm{~S})-\mathrm{C}(1 \mathrm{~S})-\mathrm{H}(1 \mathrm{SB}) & 109.5 \\ \mathrm{~N}(2)-\mathrm{C}(25)-\mathrm{H}(25 \mathrm{~A}) & 126.1 & \mathrm{H}(1 \mathrm{SA})-\mathrm{C}(1 \mathrm{~S})-\mathrm{H}(1 \mathrm{SB}) & 108.1 \\ \mathrm{~N}(2)-\mathrm{C}(26)-\mathrm{H}(26 \mathrm{~A}) & 109.5 & \mathrm{~F}(2)-\mathrm{B}(1)-\mathrm{F}(1) & 107.5(5) \\ \mathrm{N}(2)-\mathrm{C}(26)-\mathrm{H}(26 \mathrm{~B}) & 109.5 & \mathrm{~F}(2)-\mathrm{B}(1)-\mathrm{F}(4) & 109.6(6) \\ \mathrm{H}(26 \mathrm{~A})-\mathrm{C}(26)-\mathrm{H}(26 \mathrm{~B}) & 109.5 & \mathrm{~F}(1)-\mathrm{B}(1)-\mathrm{F}(4) & 109.0(6) \\ \mathrm{N}(2)-\mathrm{C}(26)-\mathrm{H}(26 \mathrm{C}) & 109.5 & \mathrm{~F}(2)-\mathrm{B}(1)-\mathrm{F}(3) & 110.8(6) \\ \mathrm{H}(26 \mathrm{~A})-\mathrm{C}(26)-\mathrm{H}(26 \mathrm{C}) & 109.5 & \mathrm{~F}(1)-\mathrm{B}(1)-\mathrm{F}(3) & 11.7(5) \\ \mathrm{H}(26 \mathrm{~B})-\mathrm{C}(26)-\mathrm{H}(26 \mathrm{C}) & 109.5 & \mathrm{~F}(4)-\mathrm{B}(1)-\mathrm{F}(3) & 108.3(5)\end{array}$


Table S9. Bond lengths $[\AA]$ and angles [deg] for $\mathbf{4 b}$.

\begin{tabular}{|c|c|c|c|}
\hline $\mathrm{Ru}(1)-\mathrm{C}(1)$ & $2.159(5)$ & $\mathrm{B}(1)-\mathrm{F}(2)$ & $1.381(5)$ \\
\hline $\mathrm{Ru}(1)-\mathrm{C}(2)$ & $2.174(5)$ & $\mathrm{B}(1)-\mathrm{F}(3)$ & $1.382(5)$ \\
\hline $\mathrm{Ru}(1)-\mathrm{C}(5)$ & $2.175(5)$ & $\mathrm{B}(1)-\mathrm{F}(1)$ & $1.387(5)$ \\
\hline $\mathrm{Ru}(1)-\mathrm{C}(6)$ & $2.177(5)$ & $\mathrm{B}(1)-\mathrm{F}(4)$ & $1.395(5)$ \\
\hline $\mathrm{Ru}(1)-\mathrm{C}(3)$ & $2.219(4)$ & & \\
\hline $\mathrm{Ru}(1)-\mathrm{C}(4)$ & $2.220(5)$ & $\mathrm{C}(1)-\mathrm{Ru}(1)-\mathrm{C}(2)$ & $37.3(2)$ \\
\hline $\mathrm{Ru}(1)-\mathrm{P}(1)$ & $2.3404(14)$ & $\mathrm{C}(1)-\mathrm{Ru}(1)-\mathrm{C}(5)$ & $67.1(2)$ \\
\hline $\mathrm{Ru}(1)-\mathrm{Cl}(1)$ & $2.3982(13)$ & $\mathrm{C}(2)-\mathrm{Ru}(1)-\mathrm{C}(5)$ & $78.88(19)$ \\
\hline $\mathrm{Ru}(1)-\mathrm{Cl}(2)$ & $2.4221(15)$ & $\mathrm{C}(1)-\mathrm{Ru}(1)-\mathrm{C}(6)$ & $37.9(2)$ \\
\hline $\mathrm{P}(1)-\mathrm{C}(13)$ & $1.805(4)$ & $\mathrm{C}(2)-\mathrm{Ru}(1)-\mathrm{C}(6)$ & $67.6(2)$ \\
\hline $\mathrm{P}(1)-\mathrm{C}(7)$ & $1.830(4)$ & $\mathrm{C}(5)-\mathrm{Ru}(1)-\mathrm{C}(6)$ & $36.9(2)$ \\
\hline $\mathrm{P}(1)-\mathrm{C}(19)$ & $1.841(4)$ & $\mathrm{C}(1)-\mathrm{Ru}(1)-\mathrm{C}(3)$ & $66.60(19)$ \\
\hline $\mathrm{N}(1)-\mathrm{C}(19)$ & $1.336(5)$ & $\mathrm{C}(2)-\mathrm{Ru}(1)-\mathrm{C}(3)$ & $36.95(18)$ \\
\hline $\mathrm{N}(1)-\mathrm{C}(21)$ & $1.363(5)$ & $\mathrm{C}(5)-\mathrm{Ru}(1)-\mathrm{C}(3)$ & $65.7(2)$ \\
\hline $\mathrm{N}(2)-\mathrm{C}(19)$ & $1.340(5)$ & $\mathrm{C}(6)-\mathrm{Ru}(1)-\mathrm{C}(3)$ & $78.60(18)$ \\
\hline $\mathrm{N}(2)-\mathrm{C}(20)$ & $1.374(5)$ & $\mathrm{C}(1)-\mathrm{Ru}(1)-\mathrm{C}(4)$ & $78.73(18)$ \\
\hline $\mathrm{N}(2)-\mathrm{C}(22)$ & $1.460(6)$ & $\mathrm{C}(2)-\mathrm{Ru}(1)-\mathrm{C}(4)$ & $65.9(2)$ \\
\hline $\mathrm{C}(1)-\mathrm{C}(2)$ & $1.386(7)$ & $\mathrm{C}(5)-\mathrm{Ru}(1)-\mathrm{C}(4)$ & $37.25(19)$ \\
\hline$C(1)-C(6)$ & $1.410(8)$ & $\mathrm{C}(6)-\mathrm{Ru}(1)-\mathrm{C}(4)$ & $66.84(19)$ \\
\hline$C(2)-C(3)$ & $1.393(7)$ & $\mathrm{C}(3)-\mathrm{Ru}(1)-\mathrm{C}(4)$ & $35.58(18)$ \\
\hline$C(3)-C(4)$ & $1.357(7)$ & $\mathrm{C}(1)-\mathrm{Ru}(1)-\mathrm{P}(1)$ & $89.43(13)$ \\
\hline$C(4)-C(5)$ & $1.404(7)$ & $\mathrm{C}(2)-\mathrm{Ru}(1)-\mathrm{P}(1)$ & $110.50(16)$ \\
\hline$C(5)-C(6)$ & $1.376(7)$ & $\mathrm{C}(5)-\mathrm{Ru}(1)-\mathrm{P}(1)$ & $127.56(17)$ \\
\hline $\mathrm{C}(7)-\mathrm{C}(8)$ & $1.380(5)$ & $\mathrm{C}(6)-\mathrm{Ru}(1)-\mathrm{P}(1)$ & $97.06(15)$ \\
\hline$C(7)-C(12)$ & $1.405(5)$ & $\mathrm{C}(3)-\mathrm{Ru}(1)-\mathrm{P}(1)$ & $146.49(15)$ \\
\hline $\mathrm{C}(8)-\mathrm{C}(9)$ & $1.383(5)$ & $\mathrm{C}(4)-\mathrm{Ru}(1)-\mathrm{P}(1)$ & $163.82(14)$ \\
\hline $\mathrm{C}(9)-\mathrm{C}(10)$ & $1.386(6)$ & $\mathrm{C}(1)-\mathrm{Ru}(1)-\mathrm{Cl}(1)$ & $124.6(2)$ \\
\hline $\mathrm{C}(10)-\mathrm{C}(11)$ & $1.362(6)$ & $\mathrm{C}(2)-\mathrm{Ru}(1)-\mathrm{Cl}(1)$ & $93.80(15)$ \\
\hline $\mathrm{C}(11)-\mathrm{C}(12)$ & $1.392(6)$ & $\mathrm{C}(5)-\mathrm{Ru}(1)-\mathrm{Cl}(1)$ & $144.48(17)$ \\
\hline $\mathrm{C}(13)-\mathrm{C}(14)$ & $1.381(5)$ & $\mathrm{C}(6)-\mathrm{Ru}(1)-\mathrm{Cl}(1)$ & $161.38(16)$ \\
\hline $\mathrm{C}(13)-\mathrm{C}(18)$ & $1.395(5)$ & $\mathrm{C}(3)-\mathrm{Ru}(1)-\mathrm{Cl}(1)$ & $87.48(14)$ \\
\hline$C(14)-C(15)$ & $1.376(5)$ & $\mathrm{C}(4)-\mathrm{Ru}(1)-\mathrm{Cl}(1)$ & $108.07(15)$ \\
\hline$C(15)-C(16)$ & $1.369(6)$ & $\mathrm{P}(1)-\mathrm{Ru}(1)-\mathrm{Cl}(1)$ & $87.70(5)$ \\
\hline $\mathrm{C}(16)-\mathrm{C}(17)$ & $1.382(6)$ & $\mathrm{C}(1)-\mathrm{Ru}(1)-\mathrm{Cl}(2)$ & $148.3(2)$ \\
\hline $\mathrm{C}(17)-\mathrm{C}(18)$ & $1.384(6)$ & $\mathrm{C}(2)-\mathrm{Ru}(1)-\mathrm{Cl}(2)$ & $158.54(16)$ \\
\hline $\mathrm{C}(20)-\mathrm{C}(21)$ & $1.334(6)$ & $\mathrm{C}(5)-\mathrm{Ru}(1)-\mathrm{Cl}(2)$ & $87.97(15)$ \\
\hline
\end{tabular}




\begin{tabular}{|c|c|c|c|}
\hline $\mathrm{C}(6)-\mathrm{Ru}(1)-\mathrm{Cl}(2)$ & $110.74(18)$ & $C(5)-C(6)-C(1)$ & $118.6(5)$ \\
\hline $\mathrm{C}(3)-\mathrm{Ru}(1)-\mathrm{Cl}(2)$ & $121.86(15)$ & $\mathrm{C}(5)-\mathrm{C}(6)-\mathrm{Ru}(1)$ & $71.5(3)$ \\
\hline $\mathrm{C}(4)-\mathrm{Ru}(1)-\mathrm{Cl}(2)$ & $93.39(14)$ & $\mathrm{C}(1)-\mathrm{C}(6)-\mathrm{Ru}(1)$ & $70.4(3)$ \\
\hline $\mathrm{P}(1)-\mathrm{Ru}(1)-\mathrm{Cl}(2)$ & $90.96(4)$ & $\mathrm{C}(8)-\mathrm{C}(7)-\mathrm{C}(12)$ & $119.5(4)$ \\
\hline $\mathrm{Cl}(1)-\mathrm{Ru}(1)-\mathrm{Cl}(2)$ & $87.08(5)$ & $\mathrm{C}(8)-\mathrm{C}(7)-\mathrm{P}(1)$ & $118.5(3)$ \\
\hline $\mathrm{C}(13)-\mathrm{P}(1)-\mathrm{C}(7)$ & 108.60(17) & $\mathrm{C}(12)-\mathrm{C}(7)-\mathrm{P}(1)$ & $121.8(3)$ \\
\hline $\mathrm{C}(13)-\mathrm{P}(1)-\mathrm{C}(19)$ & $102.41(18)$ & $\mathrm{C}(7)-\mathrm{C}(8)-\mathrm{C}(9)$ & $120.7(4)$ \\
\hline $\mathrm{C}(7)-\mathrm{P}(1)-\mathrm{C}(19)$ & $100.57(16)$ & $\mathrm{C}(8)-\mathrm{C}(9)-\mathrm{C}(10)$ & $119.7(4)$ \\
\hline $\mathrm{C}(13)-\mathrm{P}(1)-\mathrm{Ru}(1)$ & $111.47(12)$ & $\mathrm{C}(11)-\mathrm{C}(10)-\mathrm{C}(9)$ & $120.1(4)$ \\
\hline $\mathrm{C}(7)-\mathrm{P}(1)-\mathrm{Ru}(1)$ & 117.87(13) & $\mathrm{C}(10)-\mathrm{C}(11)-\mathrm{C}(12)$ & $121.1(4)$ \\
\hline $\mathrm{C}(19)-\mathrm{P}(1)-\mathrm{Ru}(1)$ & $114.37(12)$ & $C(11)-C(12)-C(7)$ & $118.8(4)$ \\
\hline $\mathrm{C}(19)-\mathrm{N}(1)-\mathrm{C}(21)$ & $109.9(4)$ & $\mathrm{C}(14)-\mathrm{C}(13)-\mathrm{C}(18)$ & $118.8(4)$ \\
\hline $\mathrm{C}(19)-\mathrm{N}(2)-\mathrm{C}(20)$ & $108.7(3)$ & $\mathrm{C}(14)-\mathrm{C}(13)-\mathrm{P}(1)$ & $123.5(3)$ \\
\hline $\mathrm{C}(19)-\mathrm{N}(2)-\mathrm{C}(22)$ & $130.2(4)$ & $\mathrm{C}(18)-\mathrm{C}(13)-\mathrm{P}(1)$ & $117.6(3)$ \\
\hline $\mathrm{C}(20)-\mathrm{N}(2)-\mathrm{C}(22)$ & $121.0(4)$ & $\mathrm{C}(15)-\mathrm{C}(14)-\mathrm{C}(13)$ & $120.9(4)$ \\
\hline$C(2)-C(1)-C(6)$ & $120.0(5)$ & $\mathrm{C}(16)-\mathrm{C}(15)-\mathrm{C}(14)$ & $120.3(4)$ \\
\hline $\mathrm{C}(2)-\mathrm{C}(1)-\mathrm{Ru}(1)$ & $71.9(3)$ & $\mathrm{C}(15)-\mathrm{C}(16)-\mathrm{C}(17)$ & $119.8(4)$ \\
\hline $\mathrm{C}(6)-\mathrm{C}(1)-\mathrm{Ru}(1)$ & $71.7(3)$ & $\mathrm{C}(16)-\mathrm{C}(17)-\mathrm{C}(18)$ & $120.2(4)$ \\
\hline $\mathrm{C}(1)-\mathrm{C}(2)-\mathrm{C}(3)$ & $119.8(5)$ & $\mathrm{C}(17)-\mathrm{C}(18)-\mathrm{C}(13)$ & $119.9(4)$ \\
\hline $\mathrm{C}(1)-\mathrm{C}(2)-\mathrm{Ru}(1)$ & $70.8(3)$ & $\mathrm{N}(1)-\mathrm{C}(19)-\mathrm{N}(2)$ & $106.8(3)$ \\
\hline $\mathrm{C}(3)-\mathrm{C}(2)-\mathrm{Ru}(1)$ & $73.3(3)$ & $\mathrm{N}(1)-\mathrm{C}(19)-\mathrm{P}(1)$ & 121.1(3) \\
\hline $\mathrm{C}(4)-\mathrm{C}(3)-\mathrm{C}(2)$ & $120.8(5)$ & $\mathrm{N}(2)-\mathrm{C}(19)-\mathrm{P}(1)$ & $132.2(3)$ \\
\hline $\mathrm{C}(4)-\mathrm{C}(3)-\mathrm{Ru}(1)$ & $72.2(3)$ & $\mathrm{C}(21)-\mathrm{C}(20)-\mathrm{N}(2)$ & $107.7(4)$ \\
\hline $\mathrm{C}(2)-\mathrm{C}(3)-\mathrm{Ru}(1)$ & $69.7(3)$ & $\mathrm{C}(20)-\mathrm{C}(21)-\mathrm{N}(1)$ & $106.9(4)$ \\
\hline $\mathrm{C}(3)-\mathrm{C}(4)-\mathrm{C}(5)$ & $119.5(5)$ & $\mathrm{F}(2)-\mathrm{B}(1)-\mathrm{F}(3)$ & $109.7(4)$ \\
\hline $\mathrm{C}(3)-\mathrm{C}(4)-\mathrm{Ru}(1)$ & $72.2(3)$ & $\mathrm{F}(2)-\mathrm{B}(1)-\mathrm{F}(1)$ & $110.3(3)$ \\
\hline $\mathrm{C}(5)-\mathrm{C}(4)-\mathrm{Ru}(1)$ & $69.6(3)$ & $\mathrm{F}(3)-\mathrm{B}(1)-\mathrm{F}(1)$ & 109.7(4) \\
\hline$C(6)-C(5)-C(4)$ & $121.1(5)$ & $\mathrm{F}(2)-\mathrm{B}(1)-\mathrm{F}(4)$ & $109.6(4)$ \\
\hline $\mathrm{C}(6)-\mathrm{C}(5)-\mathrm{Ru}(1)$ & $71.6(3)$ & $\mathrm{F}(3)-\mathrm{B}(1)-\mathrm{F}(4)$ & $109.6(3)$ \\
\hline$C(4)-C(5)-R u(1)$ & $73.1(3)$ & $\mathrm{F}(1)-\mathrm{B}(1)-\mathrm{F}(4)$ & $108.1(4$ \\
\hline
\end{tabular}


Table S10. Anisotropic displacement parameters $\left(\AA^{2} \times 10^{3}\right)$ for 1a. The anisotropic displacement factor exponent takes the form:

$$
-2 \pi^{2}\left[h^{2} a^{* 2} U 11+\ldots+2 h k a * b * U 12\right]
$$

\begin{tabular}{|c|c|c|c|c|c|c|}
\hline & U11 & U22 & U33 & U23 & U13 & U12 \\
\hline $\mathrm{Ru}(1)$ & $16(1)$ & $20(1)$ & $17(1)$ & $-2(1)$ & $5(1)$ & $0(1)$ \\
\hline $\mathrm{Cl}(1)$ & $27(1)$ & $21(1)$ & $30(1)$ & $-4(1)$ & $7(1)$ & $-2(1)$ \\
\hline $\mathrm{Cl}(2)$ & $20(1)$ & $32(1)$ & $26(1)$ & $2(1)$ & $10(1)$ & $1(1)$ \\
\hline $\mathrm{P}(1)$ & $17(1)$ & $18(1)$ & $18(1)$ & $-2(1)$ & $5(1)$ & $-1(1)$ \\
\hline $\mathrm{N}(1)$ & $20(1)$ & $30(1)$ & $24(1)$ & $-1(1)$ & $6(1)$ & $-5(1)$ \\
\hline $\mathrm{N}(2)$ & $28(1)$ & $21(1)$ & $30(1)$ & $-2(1)$ & $8(1)$ & $-4(1)$ \\
\hline $\mathrm{C}(1)$ & $20(1)$ & $24(1)$ & $25(1)$ & $1(1)$ & $1(1)$ & $2(1)$ \\
\hline $\mathrm{C}(2)$ & $23(1)$ & $33(2)$ & $21(1)$ & $-1(1)$ & $-1(1)$ & $3(1)$ \\
\hline$C(3)$ & $25(1)$ & $32(2)$ & $23(2)$ & $6(1)$ & $8(1)$ & $3(1)$ \\
\hline $\mathrm{C}(4)$ & $20(1)$ & $24(1)$ & $34(2)$ & $6(1)$ & $5(1)$ & $4(1)$ \\
\hline$C(5)$ & $19(1)$ & 21(1) & $30(2)$ & $0(1)$ & $2(1)$ & $4(1)$ \\
\hline$C(6)$ & $16(1)$ & $26(1)$ & $27(2)$ & $-1(1)$ & $7(1)$ & $4(1)$ \\
\hline$C(7)$ & $20(1)$ & $28(1)$ & $33(2)$ & $-6(1)$ & $2(1)$ & $-3(1)$ \\
\hline $\mathrm{C}(8)$ & $24(2)$ & $43(2)$ & $49(2)$ & $0(2)$ & $-3(2)$ & $-5(2)$ \\
\hline $\mathrm{C}(9)$ & $39(2)$ & $51(2)$ & $40(2)$ & $2(2)$ & $5(2)$ & $-23(2)$ \\
\hline$C(10)$ & $28(2)$ & $30(2)$ & $45(2)$ & $6(2)$ & $5(2)$ & $-7(1)$ \\
\hline $\mathrm{C}(11)$ & $19(1)$ & $25(1)$ & $17(1)$ & $-1(1)$ & $3(1)$ & $-5(1)$ \\
\hline $\mathrm{C}(12)$ & $28(2)$ & $38(2)$ & $27(2)$ & $-2(1)$ & $6(1)$ & $-15(1)$ \\
\hline $\mathrm{C}(13)$ & $38(2)$ & $25(1)$ & $34(2)$ & $-4(1)$ & $10(1)$ & $-9(1)$ \\
\hline $\mathrm{C}(14)$ & $19(1)$ & $38(2)$ & $42(2)$ & $3(2)$ & $5(1)$ & $0(1)$ \\
\hline$C(15)$ & $19(1)$ & $24(1)$ & $19(1)$ & $1(1)$ & $4(1)$ & $0(1)$ \\
\hline$C(16)$ & $26(1)$ & $25(1)$ & $21(1)$ & $-2(1)$ & $7(1)$ & $-2(1)$ \\
\hline $\mathrm{C}(17)$ & $36(2)$ & $33(2)$ & $23(2)$ & $-3(1)$ & $12(1)$ & $7(1)$ \\
\hline $\mathrm{C}(18)$ & $26(1)$ & $40(2)$ & $25(1)$ & $7(1)$ & $13(1)$ & $4(1)$ \\
\hline$C(19)$ & $24(1)$ & $40(2)$ & $28(2)$ & $2(1)$ & $8(1)$ & $-6(1)$ \\
\hline $\mathrm{C}(20)$ & $25(1)$ & $29(1)$ & $27(1)$ & $-3(1)$ & $6(1)$ & $-6(1)$ \\
\hline $\mathrm{C}(21)$ & $17(1)$ & $19(1)$ & $25(1)$ & $2(1)$ & $3(1)$ & $-2(1)$ \\
\hline $\mathrm{C}(22)$ & $27(2)$ & $24(1)$ & $28(2)$ & $-1(1)$ & $9(1)$ & $1(1)$ \\
\hline $\mathrm{C}(23)$ & $33(2)$ & $27(2)$ & $45(2)$ & $-3(1)$ & $6(2)$ & $4(1)$ \\
\hline $\mathrm{C}(24)$ & $44(2)$ & $25(2)$ & $54(2)$ & $7(2)$ & $-8(2)$ & $4(2)$ \\
\hline $\mathrm{C}(25)$ & $47(2)$ & $29(2)$ & $36(2)$ & $6(1)$ & $-9(2)$ & $-1(1)$ \\
\hline$C(26)$ & $33(2)$ & $27(1)$ & $26(2)$ & $2(1)$ & 1(1) & $-1(1)$ \\
\hline $\mathrm{C}(1 \mathrm{~S})$ & $42(3)$ & $39(2)$ & $31(2)$ & 0 & $15(2)$ & 0 \\
\hline $\mathrm{Cl}(1 \mathrm{~S})$ & $64(1)$ & $95(1)$ & $81(1)$ & $0(1)$ & $7(1)$ & $-41(1)$ \\
\hline
\end{tabular}


Table S11. Anisotropic displacement parameters $\left(\AA^{2} \times 10^{3}\right)$ for $\mathbf{1 b}$. The anisotropic displacement factor exponent takes the form:

$$
-2 \pi^{2}\left[h^{2} a^{* 2} U 11+\ldots+2 h k a * b * U 12\right]
$$

\begin{tabular}{|c|c|c|c|c|c|c|}
\hline & U11 & U22 & U33 & U23 & U13 & U12 \\
\hline $\mathrm{Ru}(1)$ & $32(1)$ & $32(1)$ & $36(1)$ & $-2(1)$ & $22(1)$ & $-2(1)$ \\
\hline $\mathrm{Cl}(1)$ & $53(1)$ & $42(1)$ & $51(1)$ & $0(1)$ & $36(1)$ & $-9(1)$ \\
\hline $\mathrm{Cl}(2)$ & $37(1)$ & $55(1)$ & $40(1)$ & $2(1)$ & $21(1)$ & $8(1)$ \\
\hline $\mathrm{P}(1)$ & $30(1)$ & $33(1)$ & $34(1)$ & $2(1)$ & $20(1)$ & $0(1)$ \\
\hline $\mathrm{N}(1)$ & $41(2)$ & $46(2)$ & $53(2)$ & $9(2)$ & $33(2)$ & $2(2)$ \\
\hline $\mathrm{N}(2)$ & $34(2)$ & $53(2)$ & $52(2)$ & $3(2)$ & $27(2)$ & $-5(2)$ \\
\hline $\mathrm{C}(1)$ & $102(4)$ & $28(2)$ & $63(3)$ & $-6(2)$ & $43(3)$ & $-4(3)$ \\
\hline $\mathrm{C}(2)$ & $47(3)$ & $58(3)$ & $75(4)$ & $-30(3)$ & $32(3)$ & $-1(2)$ \\
\hline$C(3)$ & $77(4)$ & $58(3)$ & $62(3)$ & $-30(3)$ & $51(3)$ & $-20(3)$ \\
\hline C(4) & $68(3)$ & $51(3)$ & $41(3)$ & $-13(2)$ & $25(3)$ & $-5(2)$ \\
\hline$C(5)$ & $42(3)$ & $60(3)$ & $81(4)$ & $-37(3)$ & $31(3)$ & $-16(2)$ \\
\hline$C(6)$ & $98(4)$ & $53(3)$ & $96(4)$ & $-38(3)$ & $79(4)$ & $-45(3)$ \\
\hline$C(7)$ & $43(2)$ & $35(2)$ & $40(2)$ & $1(2)$ & $29(2)$ & $0(2)$ \\
\hline$C(8)$ & $66(3)$ & $51(3)$ & $68(3)$ & $2(3)$ & $51(3)$ & $-12(2)$ \\
\hline $\mathrm{C}(9)$ & $45(3)$ & $67(3)$ & $64(3)$ & $2(3)$ & $37(3)$ & $-11(2)$ \\
\hline$C(10)$ & $55(3)$ & $78(3)$ & $75(4)$ & $35(3)$ & $41(3)$ & $16(3)$ \\
\hline$C(11)$ & $35(2)$ & $38(2)$ & $37(2)$ & $3(2)$ & $22(2)$ & $4(2)$ \\
\hline$C(12)$ & $51(3)$ & $55(3)$ & $46(3)$ & $-4(2)$ & $35(2)$ & $-3(2)$ \\
\hline$C(13)$ & $73(3)$ & $56(3)$ & $48(3)$ & $0(2)$ & $41(3)$ & $6(3)$ \\
\hline $\mathrm{C}(14)$ & $56(3)$ & $47(3)$ & $41(3)$ & $-5(2)$ & $20(2)$ & $8(2)$ \\
\hline$C(15)$ & $39(2)$ & $49(3)$ & $56(3)$ & $-12(2)$ & $24(2)$ & $-9(2)$ \\
\hline$C(16)$ & $37(2)$ & $43(2)$ & $41(2)$ & $-3(2)$ & $24(2)$ & $-2(2)$ \\
\hline$C(17)$ & $29(2)$ & $35(2)$ & $42(2)$ & $4(2)$ & $20(2)$ & $-1(2)$ \\
\hline$C(18)$ & $38(2)$ & $40(2)$ & $58(3)$ & $5(2)$ & $29(2)$ & $2(2)$ \\
\hline$C(19)$ & $42(3)$ & $39(3)$ & $87(4)$ & $3(2)$ & $38(3)$ & $3(2)$ \\
\hline$C(20)$ & $40(3)$ & $51(3)$ & $69(4)$ & $14(3)$ & $16(3)$ & $9(2)$ \\
\hline $\mathrm{C}(21)$ & $58(3)$ & $54(3)$ & $46(3)$ & $14(2)$ & $15(2)$ & $8(2)$ \\
\hline $\mathrm{C}(22)$ & $41(2)$ & $53(3)$ & $40(3)$ & $3(2)$ & $21(2)$ & $2(2)$ \\
\hline$C(1 S)$ & $67(3)$ & $90(4)$ & $63(4)$ & $-13(3)$ & $34(3)$ & $-7(3)$ \\
\hline$C(2 S)$ & $97(4)$ & $79(4)$ & $65(4)$ & $3(3)$ & $41(3)$ & $11(3)$ \\
\hline $\mathrm{Cl}(1 \mathrm{~S})$ & $90(1)$ & $127(2)$ & $129(2)$ & $-14(1)$ & $72(1)$ & $-21(1)$ \\
\hline $\mathrm{Cl}(2 \mathrm{~S})$ & $93(1)$ & $227(2)$ & $86(1)$ & $-33(2)$ & $59(1)$ & $-35(1)$ \\
\hline $\mathrm{Cl}(3 \mathrm{~S})$ & $119(1)$ & $88(1)$ & $122(2)$ & $16(1)$ & $83(1)$ & $17(1)$ \\
\hline $\mathrm{Cl}(4 \mathrm{~S})$ & $147(2)$ & $130(2)$ & $89(1)$ & $4(1)$ & $44(1)$ & $-39(2)$ \\
\hline
\end{tabular}


Table S12. Anisotropic displacement parameters $\left(\AA^{2} \times 10^{3}\right)$ for $\mathbf{4 a}$. The anisotropic displacement factor exponent takes the form:

$$
-2 \pi^{2}\left[h^{2} a^{* 2} U 11+\ldots+2 h k a^{*} b^{*} U 12\right]
$$

\begin{tabular}{|c|c|c|c|c|c|c|}
\hline & U11 & U22 & U33 & U23 & U13 & U12 \\
\hline $\mathrm{Ru}(1)$ & $16(1)$ & $16(1)$ & $22(1)$ & $1(1)$ & $1(1)$ & $-1(1)$ \\
\hline $\mathrm{P}(1)$ & $16(1)$ & $15(1)$ & $22(1)$ & $1(1)$ & $1(1)$ & 1(1) \\
\hline $\mathrm{Cl}(1)$ & $24(1)$ & $22(1)$ & $31(1)$ & $-7(1)$ & $3(1)$ & $-1(1)$ \\
\hline $\mathrm{Cl}(2)$ & $23(1)$ & $19(1)$ & $32(1)$ & $4(1)$ & $-4(1)$ & 1(1) \\
\hline $\mathrm{N}(1)$ & $18(2)$ & $20(2)$ & $36(2)$ & $2(2)$ & $2(2)$ & $-1(2)$ \\
\hline $\mathrm{N}(2)$ & $18(2)$ & $20(2)$ & $27(2)$ & $1(2)$ & $2(2)$ & $-1(2)$ \\
\hline$C(1)$ & $20(3)$ & $44(3)$ & $19(3)$ & $-2(2)$ & $4(2)$ & $-4(2)$ \\
\hline$C(2)$ & $24(3)$ & $38(3)$ & $29(3)$ & $8(2)$ & $8(2)$ & $-7(2)$ \\
\hline $\mathrm{C}(3)$ & $18(3)$ & $24(3)$ & $37(3)$ & $-2(2)$ & $0(2)$ & $-9(2)$ \\
\hline $\mathrm{C}(4)$ & $12(2)$ & $23(3)$ & $28(3)$ & $2(2)$ & $-1(2)$ & $2(2)$ \\
\hline$C(5)$ & $19(3)$ & $27(3)$ & $23(3)$ & $-1(2)$ & $7(2)$ & $1(2)$ \\
\hline$C(6)$ & $12(2)$ & $27(3)$ & $40(3)$ & $-6(2)$ & $8(2)$ & $1(2)$ \\
\hline$C(7)$ & $28(3)$ & $65(4)$ & $20(3)$ & $0(3)$ & $-3(2)$ & $-10(3)$ \\
\hline $\mathrm{C}(8)$ & $21(3)$ & $31(3)$ & $39(3)$ & $-7(3)$ & $-4(2)$ & $1(2)$ \\
\hline $\mathrm{C}(9)$ & $27(3)$ & $81(5)$ & $43(4)$ & $-24(4)$ & $-5(3)$ & $-5(3)$ \\
\hline $\mathrm{C}(10)$ & $47(4)$ & $45(4)$ & $32(3)$ & $0(3)$ & $-7(3)$ & $5(3)$ \\
\hline $\mathrm{C}(11)$ & $16(2)$ & $15(2)$ & $22(2)$ & $1(2)$ & $4(2)$ & $2(2)$ \\
\hline $\mathrm{C}(12)$ & $26(3)$ & $20(3)$ & $34(3)$ & $2(2)$ & $2(2)$ & $-2(2)$ \\
\hline $\mathrm{C}(13)$ & $33(3)$ & $16(3)$ & $39(3)$ & $-4(2)$ & $3(2)$ & $1(2)$ \\
\hline $\mathrm{C}(14)$ & $31(3)$ & $29(3)$ & $24(3)$ & $-10(2)$ & $1(2)$ & $-3(2)$ \\
\hline $\mathrm{C}(15)$ & $26(3)$ & $29(3)$ & $27(3)$ & $-1(2)$ & $-6(2)$ & $0(2)$ \\
\hline$C(16)$ & $23(3)$ & $20(3)$ & $26(3)$ & $1(2)$ & $-2(2)$ & $2(2)$ \\
\hline $\mathrm{C}(17)$ & $18(3)$ & $16(3)$ & $36(3)$ & $4(2)$ & $-1(2)$ & $-3(2)$ \\
\hline $\mathrm{C}(18)$ & $30(3)$ & $14(3)$ & $33(3)$ & $0(2)$ & $-2(2)$ & $-3(2)$ \\
\hline$C(19)$ & $37(3)$ & $21(3)$ & $37(3)$ & $5(2)$ & $-12(2)$ & $2(2)$ \\
\hline $\mathrm{C}(20)$ & $54(4)$ & $36(4)$ & $37(3)$ & $18(3)$ & $-15(3)$ & $-5(3)$ \\
\hline $\mathrm{C}(21)$ & $49(4)$ & $51(4)$ & $14(3)$ & $3(3)$ & $-1(2)$ & $-3(3)$ \\
\hline $\mathrm{C}(22)$ & $29(3)$ & $30(3)$ & $37(3)$ & $0(3)$ & $7(2)$ & $-1(2)$ \\
\hline $\mathrm{C}(23)$ & $17(2)$ & $18(2)$ & $26(3)$ & $1(2)$ & $4(2)$ & $1(2)$ \\
\hline $\mathrm{C}(24)$ & $22(3)$ & $28(3)$ & $33(3)$ & $3(2)$ & $5(2)$ & $4(2)$ \\
\hline $\mathrm{C}(25)$ & $11(2)$ & $33(3)$ & $43(3)$ & $4(3)$ & $1(2)$ & $5(2)$ \\
\hline $\mathrm{C}(26)$ & $26(3)$ & $26(3)$ & $68(4)$ & $-7(3)$ & $13(3)$ & $-12(2)$ \\
\hline $\mathrm{C}(1 \mathrm{~S})$ & $50(4)$ & $78(5)$ & $63(5)$ & $-8(4)$ & $8(3)$ & $0(4)$ \\
\hline $\mathrm{Cl}(1 \mathrm{~S})$ & $66(1)$ & $62(1)$ & $95(2)$ & $18(1)$ & $27(1)$ & $-1(1)$ \\
\hline $\mathrm{Cl}(2 \mathrm{~S})$ & $152(2)$ & $53(1)$ & $98(2)$ & $10(1)$ & $64(2)$ & $19(1)$ \\
\hline $\mathrm{B}(1)$ & $32(4)$ & $33(4)$ & $45(4)$ & $5(3)$ & $9(3)$ & $-2(3)$ \\
\hline $\mathrm{F}(1)$ & $50(2)$ & $110(4)$ & $65(3)$ & $5(3)$ & $15(2)$ & $-34(2)$ \\
\hline $\mathrm{F}(2)$ & $57(3)$ & $53(3)$ & $241(7)$ & $2(4)$ & $27(4)$ & $12(3)$ \\
\hline $\mathrm{F}(3)$ & $74(3)$ & $62(3)$ & $61(2)$ & $-13(2)$ & $-1(2)$ & $-17(2)$ \\
\hline $\mathrm{F}(4)$ & $216(6)$ & $77(3)$ & $40(3)$ & $17(2)$ & $-10(3)$ & $-34(4)$ \\
\hline
\end{tabular}


Table S13. Anisotropic displacement parameters $\left(\AA^{2} \times 10^{3}\right)$ for $\mathbf{4 b}$. The anisotropic displacement factor exponent takes the form:

$$
-2 \pi^{2}\left[h^{2} a^{* 2} U 11+\ldots+2 h k a * b * U 12\right]
$$

\begin{tabular}{|c|c|c|c|c|c|c|}
\hline & U11 & U22 & U33 & U23 & U13 & U12 \\
\hline $\mathrm{Ru}(1)$ & $20(1)$ & $20(1)$ & $13(1)$ & $0(1)$ & $1(1)$ & $-2(1)$ \\
\hline $\mathrm{Cl}(1)$ & $30(1)$ & $28(1)$ & $17(1)$ & $3(1)$ & $-2(1)$ & $2(1)$ \\
\hline $\mathrm{Cl}(2)$ & $33(1)$ & $26(1)$ & $27(1)$ & $-2(1)$ & $4(1)$ & $-11(1)$ \\
\hline $\mathrm{P}(1)$ & $20(1)$ & $17(1)$ & $16(1)$ & $2(1)$ & $2(1)$ & $-1(1)$ \\
\hline $\mathrm{N}(1)$ & $39(2)$ & $18(2)$ & $26(2)$ & $4(2)$ & $10(2)$ & $0(2)$ \\
\hline $\mathrm{N}(2)$ & $27(2)$ & $18(2)$ & $18(2)$ & $0(1)$ & $-2(2)$ & $3(2)$ \\
\hline $\mathrm{C}(1)$ & $49(4)$ & $20(3)$ & $89(5)$ & $23(3)$ & $46(4)$ & $19(3)$ \\
\hline$C(2)$ & $36(3)$ & $28(3)$ & $64(4)$ & $-17(3)$ & $-14(3)$ & $3(2)$ \\
\hline C(3) & $64(4)$ & $38(3)$ & $23(3)$ & $-3(2)$ & $6(3)$ & $22(3)$ \\
\hline$C(4)$ & $33(3)$ & $33(3)$ & $65(4)$ & $9(3)$ & 21(3) & $6(2)$ \\
\hline$C(5)$ & $33(3)$ & $38(3)$ & $71(4)$ & $-21(3)$ & $-23(3)$ & $13(3)$ \\
\hline$C(6)$ & $72(4)$ & $53(3)$ & $16(3)$ & $-1(2)$ & $3(3)$ & $35(3)$ \\
\hline$C(7)$ & $23(2)$ & $18(2)$ & $15(2)$ & $0(2)$ & $3(2)$ & $0(2)$ \\
\hline$C(8)$ & $24(3)$ & $23(2)$ & $19(2)$ & $-1(2)$ & $3(2)$ & $1(2)$ \\
\hline C(9) & $32(3)$ & $23(2)$ & $24(2)$ & $-5(2)$ & $-3(2)$ & $-3(2)$ \\
\hline$C(10)$ & $40(3)$ & $31(3)$ & $15(2)$ & $-6(2)$ & $-3(2)$ & $6(2)$ \\
\hline $\mathrm{C}(11)$ & $41(3)$ & $51(3)$ & $16(3)$ & $2(2)$ & $12(2)$ & $5(3)$ \\
\hline$C(12)$ & $23(3)$ & $45(3)$ & $23(2)$ & $-1(2)$ & $3(2)$ & $-1(2)$ \\
\hline$C(13)$ & $21(2)$ & $14(2)$ & $17(2)$ & $1(2)$ & $2(2)$ & $1(2)$ \\
\hline $\mathrm{C}(14)$ & $27(3)$ & $23(2)$ & $23(2)$ & $7(2)$ & $-2(2)$ & $-2(2)$ \\
\hline$C(15)$ & $30(3)$ & $17(2)$ & $36(3)$ & $12(2)$ & $4(2)$ & $-6(2)$ \\
\hline$C(16)$ & $24(3)$ & $24(2)$ & $40(3)$ & $-4(2)$ & $3(2)$ & $-4(2)$ \\
\hline$C(17)$ & $32(3)$ & $30(3)$ & $24(3)$ & $-5(2)$ & $0(2)$ & $-3(2)$ \\
\hline$C(18)$ & $26(3)$ & $21(2)$ & $24(2)$ & $1(2)$ & $4(2)$ & $-2(2)$ \\
\hline$C(19)$ & $28(3)$ & $16(2)$ & $14(2)$ & $-3(2)$ & $-1(2)$ & $-3(2)$ \\
\hline$C(20)$ & $35(3)$ & $24(2)$ & $19(2)$ & $-1(2)$ & $-1(2)$ & $9(2)$ \\
\hline$C(21)$ & $48(3)$ & $14(2)$ & $21(2)$ & $2(2)$ & $2(2)$ & $4(2)$ \\
\hline$C(22)$ & $26(3)$ & $41(3)$ & $41(3)$ & $7(3)$ & $9(2)$ & $2(3)$ \\
\hline $\mathrm{B}(1)$ & $25(3)$ & $27(3)$ & $25(3)$ & $-1(2)$ & $3(2)$ & $0(2)$ \\
\hline $\mathrm{F}(1)$ & $37(2)$ & $41(2)$ & $26(1)$ & 1(1) & 11(1) & $5(1)$ \\
\hline $\mathrm{F}(2)$ & $31(2)$ & $28(1)$ & $50(2)$ & $7(1)$ & $2(1)$ & $6(1)$ \\
\hline $\mathrm{F}(3)$ & $42(2)$ & $29(1)$ & $65(2)$ & $10(1)$ & $21(1)$ & $-3(1)$ \\
\hline $\mathrm{F}(4)$ & $43(2)$ & $63(2)$ & $31(2)$ & $-13(1)$ & $-6(1)$ & $5(1)$ \\
\hline
\end{tabular}


Table S14. Hydrogen coordinates ( $\times 10^{4}$ ) and isotropic displacement parameters $\left(\AA^{2} \times 10^{3}\right.$ ) for $\mathbf{1 a}$.

\begin{tabular}{|c|c|c|c|c|}
\hline & $\mathrm{x}$ & $\mathrm{y}$ & $\mathrm{z}$ & $\mathrm{U}(\mathrm{eq})$ \\
\hline $\mathrm{H}(2)$ & $9480(20)$ & $2310(30)$ & $12308(19)$ & $29(8)$ \\
\hline $\mathrm{H}(3)$ & $8295(19)$ & $880(20)$ & $12324(16)$ & $17(7)$ \\
\hline $\mathrm{H}(5)$ & $8678(17)$ & $-180(20)$ & $10106(15)$ & $9(6)$ \\
\hline $\mathrm{H}(6)$ & $9910(20)$ & 1380(30) & $10152(18)$ & $29(8)$ \\
\hline $\mathrm{H}(7)$ & $10380(20)$ & $3710(30)$ & $11533(18)$ & $31(8)$ \\
\hline $\mathrm{H}(8 \mathrm{~A})$ & $11770(20)$ & $1710(30)$ & 11624(19) & $38(9)$ \\
\hline $\mathrm{H}(8 \mathrm{~B})$ & $11980(20)$ & $3110(30)$ & $11890(19)$ & $43(10)$ \\
\hline $\mathrm{H}(8 \mathrm{C})$ & $11280(30)$ & $2280(30)$ & $12310(20)$ & $47(11)$ \\
\hline $\mathrm{H}(9 \mathrm{~A})$ & $11470(20)$ & $3940(30)$ & $10618(18)$ & $33(8)$ \\
\hline $\mathrm{H}(9 \mathrm{~B})$ & $11200(30)$ & $2640(40)$ & $10260(20)$ & $63(13)$ \\
\hline $\mathrm{H}(9 \mathrm{C})$ & $10510(30)$ & $3680(30)$ & $10120(20)$ & $53(11)$ \\
\hline $\mathrm{H}(10 \mathrm{~A})$ & $7100(30)$ & $-490(30)$ & $11510(20)$ & $51(11)$ \\
\hline $\mathrm{H}(10 \mathrm{~B})$ & $7320(30)$ & $-1070(30)$ & $10720(20)$ & $55(11)$ \\
\hline $\mathrm{H}(10 \mathrm{C})$ & $7820(30)$ & $-1560(40)$ & $11540(20)$ & $69(13)$ \\
\hline $\mathrm{H}(12)$ & $4860(20)$ & $-450(30)$ & $9008(16)$ & $25(8)$ \\
\hline $\mathrm{H}(13)$ & $6340(20)$ & $-1810(30)$ & $9048(17)$ & $30(8)$ \\
\hline $\mathrm{H}(14 \mathrm{~A})$ & $5280(20)$ & $2410(30)$ & $9810(20)$ & $43(10)$ \\
\hline $\mathrm{H}(14 \mathrm{~B})$ & $5150(30)$ & $2600(30)$ & $8890(20)$ & $56(11)$ \\
\hline $\mathrm{H}(14 \mathrm{C})$ & $4470(30)$ & $1700(30)$ & $9280(20)$ & $56(11)$ \\
\hline $\mathrm{H}(16)$ & $7950(20)$ & $560(30)$ & $8256(18)$ & $39(9)$ \\
\hline $\mathrm{H}(17)$ & $9140(20)$ & $480(30)$ & $7479(18)$ & $30(8)$ \\
\hline $\mathrm{H}(18)$ & $10360(20)$ & 1940(30) & $7603(18)$ & $30(8)$ \\
\hline H(19) & $10330(30)$ & $3570(40)$ & $8520(20)$ & $59(12)$ \\
\hline $\mathrm{H}(20)$ & $9170(20)$ & $3700(30)$ & $9284(18)$ & $31(8)$ \\
\hline $\mathrm{H}(22)$ & $6660(20)$ & $4350(30)$ & $10238(18)$ & $27(8)$ \\
\hline $\mathrm{H}(23)$ & $5720(20)$ & $6040(30)$ & $9790(20)$ & 41(9) \\
\hline $\mathrm{H}(24)$ & $5410(30)$ & $6250(40)$ & $8440(20)$ & $57(11)$ \\
\hline $\mathrm{H}(25)$ & $6060(20)$ & $4950(30)$ & $7560(20)$ & $45(10)$ \\
\hline $\mathrm{H}(26)$ & $7000(20)$ & $3270(30)$ & $8065(19)$ & $34(9)$ \\
\hline $\mathrm{H}(1 \mathrm{~A})$ & $2240(20)$ & $5520(30)$ & $7890(20)$ & $49(10)$ \\
\hline
\end{tabular}


Table S15. Hydrogen coordinates ( x $\left.10^{4}\right)$ and isotropic displacement parameters $\left(\AA^{2} \times 10^{3}\right)$ for $\mathbf{1 b}$.

\begin{tabular}{|c|c|c|c|c|}
\hline & $\mathrm{x}$ & $\mathrm{y}$ & $\mathrm{z}$ & $\mathrm{U}(\mathrm{eq})$ \\
\hline $\mathrm{H}(1 \mathrm{~A})$ & 1909 & 95 & 5370 & 83 \\
\hline $\mathrm{H}(2 \mathrm{~B})$ & 2265 & 1006 & 4876 & 76 \\
\hline $\mathrm{H}(3 \mathrm{~A})$ & 1886 & 2933 & 3976 & 72 \\
\hline $\mathrm{H}(4 \mathrm{~A})$ & 1152 & 3956 & 3521 & 70 \\
\hline $\mathrm{H}(5 \mathrm{~A})$ & 785 & 3015 & 3977 & 78 \\
\hline $\mathrm{H}(6 \mathrm{~A})$ & 1161 & 1039 & 4902 & 84 \\
\hline $\mathrm{H}(8 \mathrm{~A})$ & 768 & 1207 & 6061 & 66 \\
\hline $\mathrm{H}(9 \mathrm{~A})$ & 114 & 2858 & 5149 & 68 \\
\hline $\mathrm{H}(10 \mathrm{~A})$ & 1812 & 2666 & 6323 & 103 \\
\hline $\mathrm{H}(10 \mathrm{~B})$ & 1622 & 824 & 6301 & 103 \\
\hline $\mathrm{H}(10 \mathrm{C})$ & 1696 & 2221 & 6816 & 103 \\
\hline $\mathrm{H}(12 \mathrm{~A})$ & 1356 & 5887 & 6428 & 57 \\
\hline $\mathrm{H}(13 \mathrm{~A})$ & 1805 & 7462 & 7415 & 67 \\
\hline $\mathrm{H}(14 \mathrm{~A})$ & 2409 & 9082 & 7639 & 65 \\
\hline $\mathrm{H}(15 \mathrm{~A})$ & 2577 & 9050 & 6894 & 62 \\
\hline $\mathrm{H}(16 \mathrm{~A})$ & 2165 & 7339 & 5942 & 48 \\
\hline $\mathrm{H}(18 \mathrm{~A})$ & 767 & 7760 & 5242 & 55 \\
\hline H(19A) & 198 & 9618 & 4430 & 69 \\
\hline $\mathrm{H}(20 \mathrm{~A})$ & 33 & 9822 & 3391 & 77 \\
\hline $\mathrm{H}(21 \mathrm{~A})$ & 446 & 8234 & 3146 & 76 \\
\hline $\mathrm{H}(22 \mathrm{~A})$ & 1009 & 6350 & 3944 & 57 \\
\hline $\mathrm{H}(1 \mathrm{SA})$ & 53 & 5101 & 6098 & 93 \\
\hline $\mathrm{H}(1 \mathrm{SB})$ & 527 & 4292 & 6680 & 93 \\
\hline $\mathrm{H}(2 \mathrm{SA})$ & 1924 & 1411 & 8370 & 103 \\
\hline $\mathrm{H}(2 \mathrm{SB})$ & 1666 & 1997 & 8659 & 103 \\
\hline
\end{tabular}


Table S16. Hydrogen coordinates ( x 10 $)$ and isotropic displacement parameters $\left(\AA^{2} \times 10^{3}\right)$ for $\mathbf{4 a}$.

\begin{tabular}{|c|c|c|c|c|}
\hline & $\mathrm{x}$ & $\mathrm{y}$ & $\mathrm{z}$ & $\mathrm{U}(\mathrm{eq})$ \\
\hline $\mathrm{H}(1 \mathrm{~A})$ & 9593 & 4360 & 7674 & 30 \\
\hline $\mathrm{H}(2 \mathrm{~A})$ & 6429 & 3651 & 9812 & 36 \\
\hline $\mathrm{H}(3 \mathrm{~A})$ & 5849 & 3402 & 8311 & 32 \\
\hline $\mathrm{H}(5 \mathrm{~A})$ & 5755 & 5918 & 7833 & 27 \\
\hline $\mathrm{H}(6 \mathrm{~A})$ & 6359 & 6162 & 9322 & 31 \\
\hline $\mathrm{H}(7 \mathrm{~A})$ & 6167 & 5143 & 11072 & 57 \\
\hline $\mathrm{H}(7 \mathrm{~B})$ & 7182 & 5594 & 10695 & 57 \\
\hline $\mathrm{H}(7 \mathrm{C})$ & 7200 & 4620 & 10863 & 57 \\
\hline $\mathrm{H}(8 \mathrm{~A})$ & 5573 & 3920 & 6840 & 36 \\
\hline $\mathrm{H}(9 \mathrm{~A})$ & 3873 & 3880 & 7442 & 76 \\
\hline $\mathrm{H}(9 \mathrm{~B})$ & 3801 & 4108 & 6409 & 76 \\
\hline $\mathrm{H}(9 \mathrm{C})$ & 3674 & 4814 & 7139 & 76 \\
\hline $\mathrm{H}(10 \mathrm{~A})$ & 6278 & 5181 & 6346 & 63 \\
\hline $\mathrm{H}(10 \mathrm{~B})$ & 5157 & 5617 & 6465 & 63 \\
\hline $\mathrm{H}(10 \mathrm{C})$ & 5278 & 4909 & 5736 & 63 \\
\hline $\mathrm{H}(12 \mathrm{~A})$ & 8223 & 7655 & 8308 & 32 \\
\hline $\mathrm{H}(13 \mathrm{~A})$ & 8453 & 8591 & 9483 & 35 \\
\hline $\mathrm{H}(14 \mathrm{~A})$ & 8927 & 8120 & 10899 & 34 \\
\hline $\mathrm{H}(15 \mathrm{~A})$ & 9159 & 6710 & 11155 & 33 \\
\hline $\mathrm{H}(16 \mathrm{~A})$ & 8924 & 5775 & 9993 & 28 \\
\hline $\mathrm{H}(18 \mathrm{~A})$ & 6795 & 7038 & 7643 & 31 \\
\hline $\mathrm{H}(19 \mathrm{~A})$ & 6181 & 7604 & 6311 & 38 \\
\hline $\mathrm{H}(20 \mathrm{~A})$ & 6988 & 7271 & 5004 & 51 \\
\hline $\mathrm{H}(21 \mathrm{~A})$ & 8377 & 6330 & 4998 & 46 \\
\hline $\mathrm{H}(22 \mathrm{~A})$ & 9039 & 5774 & 6353 & 38 \\
\hline $\mathrm{H}(24 \mathrm{~A})$ & 11464 & 4296 & 7316 & 33 \\
\hline $\mathrm{H}(25 \mathrm{~A})$ & 12146 & 5714 & 7564 & 35 \\
\hline $\mathrm{H}(26 \mathrm{~A})$ & 10055 & 7107 & 8310 & 59 \\
\hline $\mathrm{H}(26 \mathrm{~B})$ & 10982 & 7185 & 7620 & 59 \\
\hline $\mathrm{H}(26 \mathrm{C})$ & 11255 & 6947 & 8633 & 59 \\
\hline $\mathrm{H}(1 \mathrm{SA})$ & 6357 & 8108 & 9376 & 76 \\
\hline $\mathrm{H}(1 \mathrm{SB})$ & 5132 & 8204 & 9589 & 76 \\
\hline
\end{tabular}


Table S17. Hydrogen coordinates ( x $\left.10^{4}\right)$ and isotropic displacement parameters $\left(\AA^{2} \times 10^{3}\right)$ for $\mathbf{4 b}$.

\begin{tabular}{lcccc}
\hline & $\mathrm{x}$ & $\mathrm{y}$ & $\mathrm{z}$ & $\mathrm{U}(\mathrm{eq})$ \\
\hline $\mathrm{H}(1 \mathrm{~A})$ & $2280(40)$ & $6210(30)$ & $1180(20)$ & $42(14)$ \\
$\mathrm{H}(1 \mathrm{~B})$ & $2930(50)$ & $2050(40)$ & $1570(30)$ & $58(17)$ \\
$\mathrm{H}(2)$ & $2670(50)$ & $2140(30)$ & $310(20)$ & $50(15)$ \\
$\mathrm{H}(3)$ & $4310(40)$ & $3030(30)$ & $-300(20)$ & $42(13)$ \\
$\mathrm{H}(4)$ & $6040(50)$ & $3860(40)$ & $370(30)$ & $77(17)$ \\
$\mathrm{H}(5)$ & $4020(40)$ & $4640(30)$ & $2713(19)$ & $23(10)$ \\
$\mathrm{H}(5)$ & $6030(40)$ & $3750(30)$ & $1690(20)$ & $45(14)$ \\
$\mathrm{H}(6)$ & $4590(40)$ & $2840(30)$ & $2250(20)$ & $41(14)$ \\
$\mathrm{H}(9)$ & $4420(40)$ & $5040(30)$ & $3960(20)$ & $47(15)$ \\
$\mathrm{H}(10)$ & $2910(40)$ & $4840(30)$ & $4850(20)$ & $37(12)$ \\
$\mathrm{H}(11)$ & $830(40)$ & $4350(30)$ & $4370(20)$ & $35(12)$ \\
$\mathrm{H}(12)$ & $250(40)$ & $3990(30)$ & $3120(20)$ & $33(11)$ \\
$\mathrm{H}(14)$ & $780(30)$ & $2400(20)$ & $2565(19)$ & $16(10)$ \\
$\mathrm{H}(15)$ & $-870(30)$ & $1290(20)$ & $2264(19)$ & $16(10)$ \\
$\mathrm{H}(16)$ & $-2140(40)$ & $1370(30)$ & $1136(19)$ & $25(11)$ \\
$\mathrm{H}(17)$ & $-1770(40)$ & $2670(30)$ & $350(20)$ & $16(11)$ \\
$\mathrm{H}(18)$ & $-140(30)$ & $3830(30)$ & $585(18)$ & $17(10)$ \\
$\mathrm{H}(20)$ & $-1370(40)$ & $7000(30)$ & $1461(19)$ & $23(11)$ \\
$\mathrm{H}(21)$ & $790(30)$ & $7640(30)$ & $985(18)$ & $15(10)$ \\
$\mathrm{H}(22 \mathrm{~A})$ & $-1490(40)$ & $5330(30)$ & $2510(30)$ & $49(15)$ \\
$\mathrm{H}(22 \mathrm{~B})$ & $-1220(50)$ & $4470(40)$ & $2090(30)$ & $74(19)$ \\
$\mathrm{H}(22 \mathrm{C})$ & $-2180(50)$ & $5310(40)$ & $1810(30)$ & $55(18)$ \\
\hline & & & &
\end{tabular}


Figure S1. ORTEP drawing of 1a

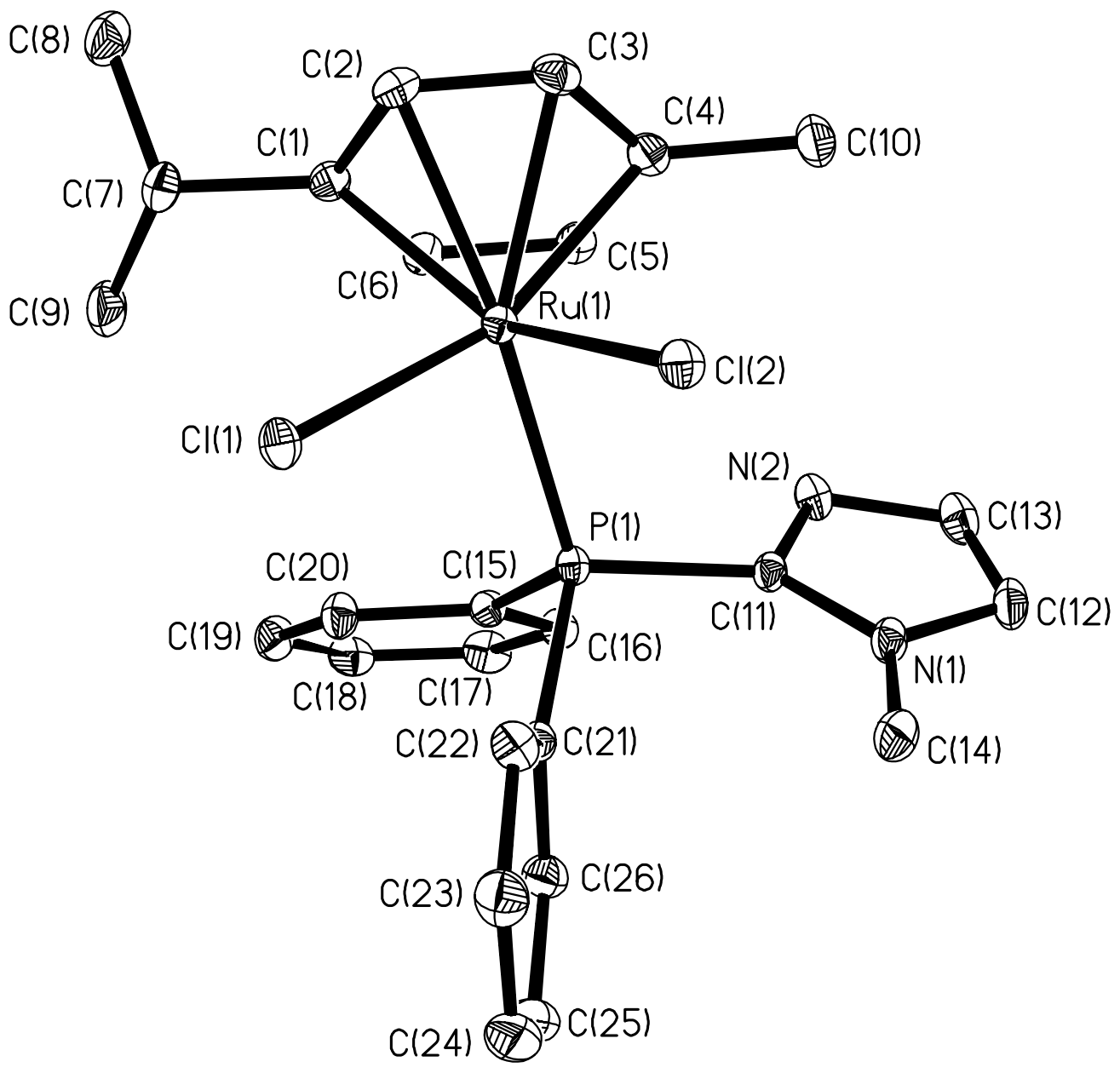


Figure S2. ORTEP drawing of $\mathbf{1 b}$

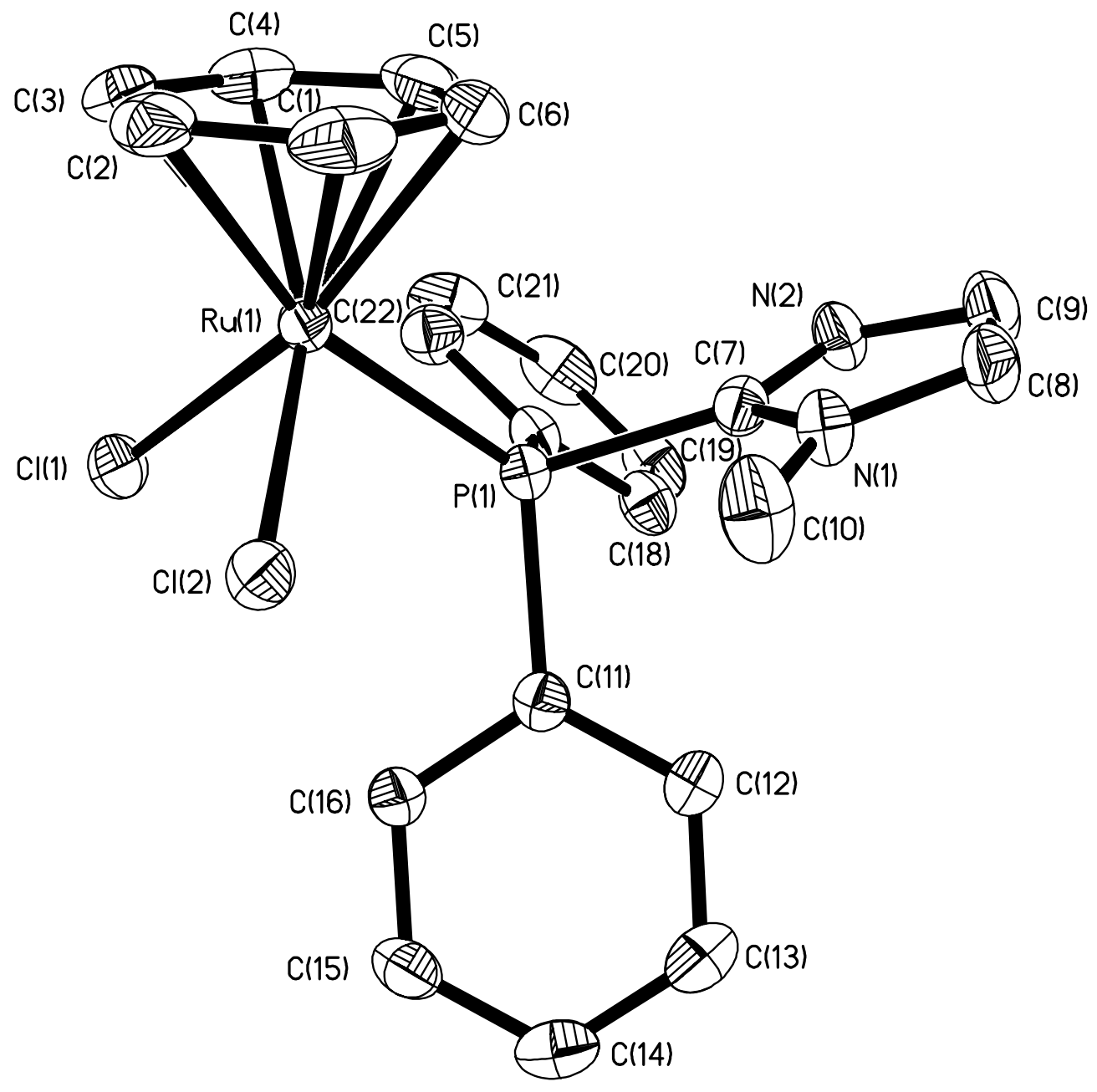


Figure S3. ORTEP drawing of $\mathbf{4 a}$

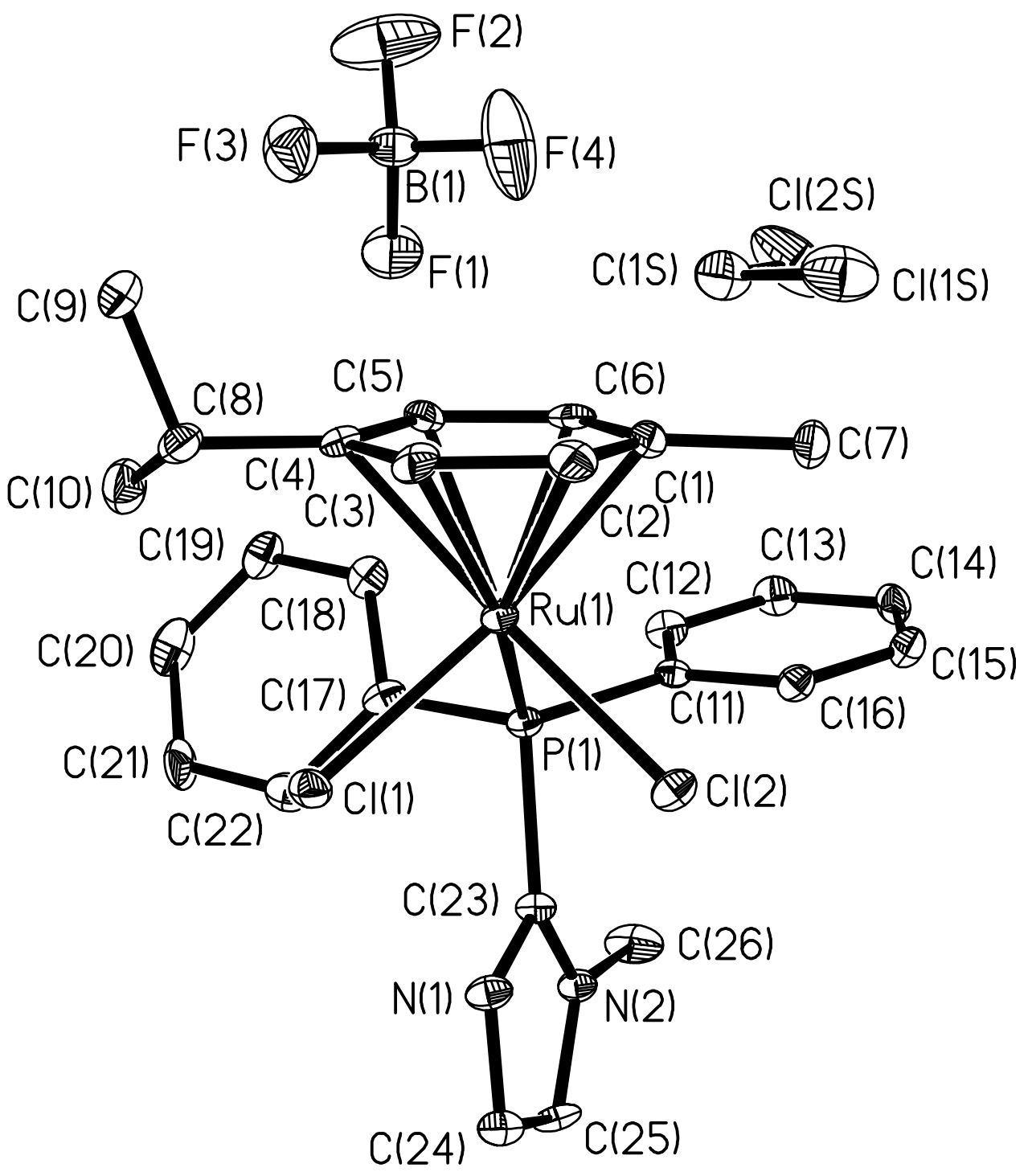


Figure S4. ORTEP drawing of $\mathbf{4 b}$
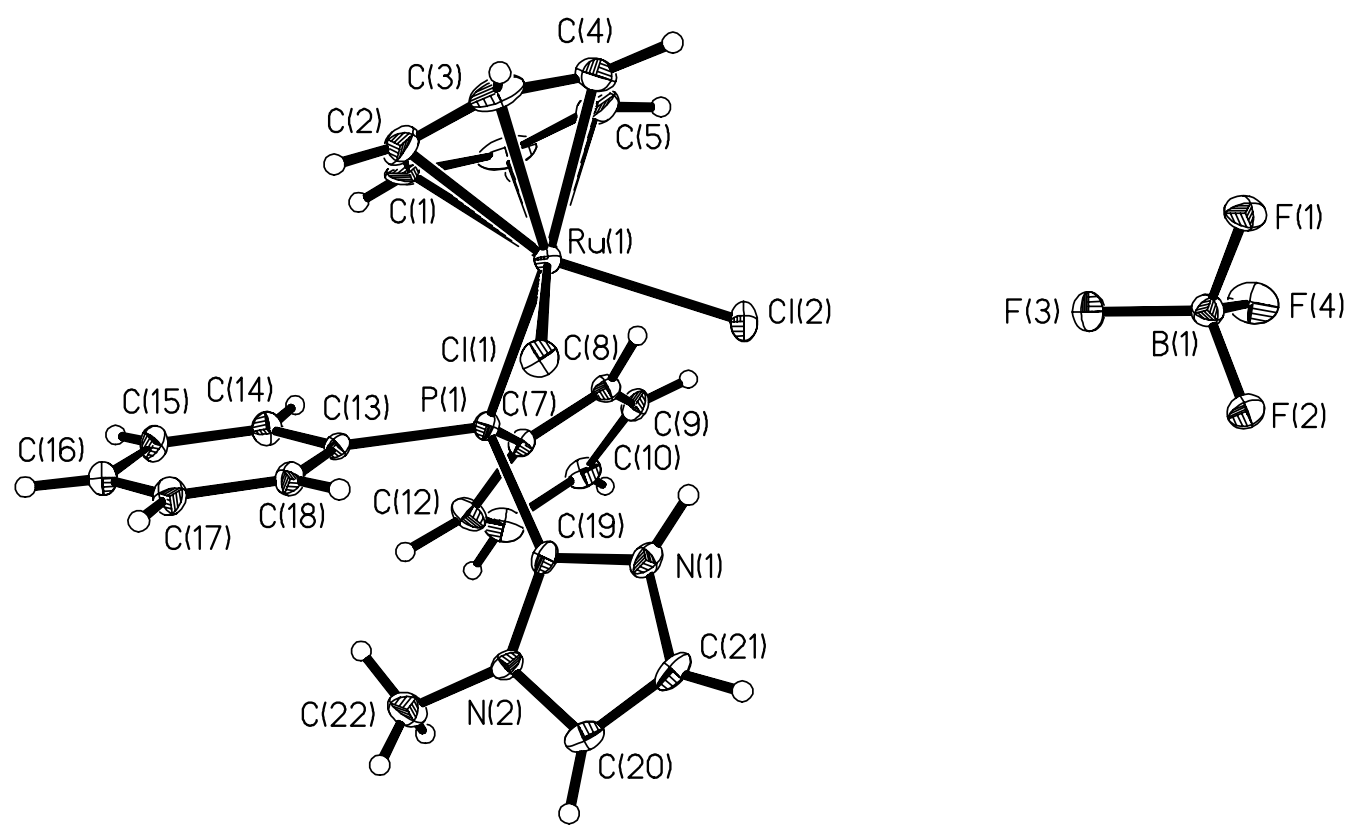